CONFORMAL GEOMETRY AND DYNAMICS

An Electronic Journal of the American Mathematical Society

Volume 12, Pages 67-96 (June 10, 2008)

S 1088-4173(08)00178-1

\title{
MÖBIUS INVARIANT METRICS BILIPSCHITZ EQUIVALENT TO THE HYPERBOLIC METRIC
}

\author{
DAVID A. HERRON, WILLIAM MA, AND DAVID MINDA
}

Dedicated to Roger Barnard on the occasion of his $65^{\text {th }}$ birthday.

\begin{abstract}
We study three Möbius invariant metrics, and three affine invariant analogs, all of which are bilipschitz equivalent to the Poincaré hyperbolic metric. We exhibit numerous illustrative examples.
\end{abstract}

\section{Contents}

1. Introduction

2. Preliminaries

3. Basic properties

4. Examples

5. Proofs of main results 90

References 95

\section{INTRODUCTION}

Precise definitions are provided in Section 2, but we mention a few items here. Everywhere below $C$ denotes a generic absolute constant. See $\$ 2 . C$ where we describe the constants

$$
k:=\frac{\Gamma^{4}(1 / 4)}{4 \pi^{2}}=4.3768796 \ldots \quad \text { and } \quad h:=\frac{\sqrt{3} \Gamma^{6}(1 / 3)}{2^{2 / 3} 4 \pi^{3}}=3.2519268 \ldots .
$$

Throughout this article $\Omega$ is a hyperbolic region in the Riemann sphere $\hat{\mathbb{C}}$; i.e., $\Omega$ is open and connected with its complement $\Omega^{c}:=\hat{\mathbb{C}} \backslash \Omega$ possessing at least three points. Each such $\Omega$ carries a unique maximal constant curvature -1 conformal metric $\lambda(z)|d z|=\lambda_{\Omega}(z)|d z|$ referred to as the Poincaré hyperbolic metric in $\Omega$. The domain monotonicity property, that larger regions have smaller metrics, is a direct consequence of Schwarz' Lemma.

Except for a short list of special cases, the actual calculation of any given hyperbolic metric is notoriously difficult. Indeed, one can find a number of papers analyzing the behavior of the hyperbolic metric in a thrice punctured sphere. Typically

Received by the editors November 30, 2007.

2000 Mathematics Subject Classification. Primary: 30F45; Secondary: 30C55, 30F30.

Key words and phrases. Möbius metrics, Poincaré hyperbolic metric, uniformly perfect.

The first and third authors were supported by the Charles Phelps Taft Research Center. 
one is left with estimates obtained by using domain monotonicity and considering 'nice' sub-domains and super-domains in which one can calculate the metric.

It is especially desirable to have both upper and lower estimates for the hyperbolic metric. In general, finding lower estimates for the hyperbolic metric seems to be the more diffcult endeavor. As is well known, the hyperbolic and quasihyperbolic metrics are 2-bilipschitz equivalent for simply connected plane hyperbolic regions; this fact is not true, e.g., for any domain with an isolated boundary point (such as the punctured unit disk), but does hold in general precisely when $\partial \Omega$ is uniformly perfect (cf. [BP78], Pom79, and Pom84]). Beardon and Pommerenke corroborated this latter assertion as an application of their elegant result BP78, Theorem 1] which says:

For any hyperbolic region $\Omega$ in $\mathbb{C}$ and for all $z \in \Omega$,

$$
\frac{1}{\delta(z)[k+\beta(z)]} \leq \lambda(z) \leq \frac{\pi}{2} \frac{1}{\delta(z) \beta(z)} .
$$

Here $\delta(z)$ denotes the distance from $z$ to the boundary of $\Omega$ (see $92 . \mathrm{A}$ ) and the domain function $\beta$ (described at the beginning of 2 .D ) was introduced by Beardon and Pommerenke.

A Möbius invariant analog of the quasihyperbolic metric $|d z| / \delta(z)$ is the so-called Ferrand metric $\varphi(z)|d z|$ (see the end of $\$ 2 . \mathrm{C}$ ). For a region $\Omega \subset \hat{\mathbb{C}}$, we define the domain function

$$
\gamma(z)=\gamma_{\Omega}(z):=\inf _{a, b, c \in \Omega^{c}}|\log | z, a, b, c||
$$

where the infimum is taken over all distinct points $a, b, c$ in the complement of $\Omega$ with $\varphi(z)=|a-b| /(|z-a||z-b|)$. Here $|z, a, b, c|:=|[z, a, b, c]|$ is the absolute cross ratio, and

$$
\text { for distinct } z, a, b, c \in \mathbb{C}, \quad[z, a, b, c]:=\frac{(z-a)(b-c)}{(z-b)(a-c)},
$$

with the usual interpretation if one of $z, a, b, c$ is the point at infinity. One can think of $\gamma$ as a Möbius invariant analog of $\beta$. In $\$ 3$. B we provide a geometric interpretation for $\gamma$.

We establish the following Möbius invariant analog of [BP78, Theorem 1].

Theorem A. For any hyperbolic region $\Omega$ in $\hat{\mathbb{C}}$ and for all $z \in \Omega$,

$$
\frac{\varphi(z)}{k+\gamma(z)} \leq \lambda(z) \leq \frac{\pi}{2} \frac{\varphi(z)}{\gamma(z)}
$$

One upshot of the above is that the hyperbolic metric $\lambda(z)|d z|$ is bilipschitz equivalent to the Möbius invariant metric $\alpha(z)|d z|=\alpha_{\Omega}(z)|d z|$ defined via $\alpha(z)=$ $\alpha_{\Omega}(z):=\varphi(z) /[k+\gamma(z)] ;$ see $₫ 2 . \mathrm{D}$.

Corollary B. For any hyperbolic region $\Omega$ in $\hat{\mathbb{C}}$, the metrics $\alpha(z)|d z|$ and $\lambda(z)|d z|$ satisfy

$$
\alpha \leq \lambda \leq C \alpha
$$

The smallest such absolute constant $C$ satisfies $k \leq C \leq k+\pi / 2$. For simply connected regions we can take $C=k$. 
Our interest in this research was initiated by work of Gardiner and Lakic GL01] and Sugawa and Vuorinen SV05. We define the Gardiner-Lakic metric $\kappa(z)|d z|=$ $\kappa_{\Omega}(z)|d z|$ via

$$
\kappa(z)=\kappa_{\Omega}(z):=\sup _{a, b, c \in \Omega^{c}} \lambda_{a b c}(z)
$$

for $z \in \Omega \cap \mathbb{C}$ and for distinct points $a, b, c \in \hat{\mathbb{C}}, \lambda_{a b c}(z)|d z|$ denotes the hyperbolic metric in the thrice punctured sphere $\hat{\mathbb{C}}_{a b c}:=\hat{\mathbb{C}} \backslash\{a, b, c\}$. Since $\hat{\mathbb{C}}_{a b c} \supset \Omega$ when $a, b, c \in \Omega^{c}$, domain monotonicity tells us that $\lambda_{a b c} \leq \lambda$; thus $\kappa \leq \lambda$. Gardiner and Lakic proved that there is an absolute constant $C$ with $\kappa \leq \lambda \leq C \kappa$ always holding GL01, Theorem 3]. However, they provided no actual value for such a $C$. As an application of Theorem $\mathrm{A}$, we deduce the following.

Corollary C. For any hyperbolic region $\Omega$ in $\hat{\mathbb{C}}$, the metrics $\kappa(z)|d z|$ and $\lambda(z)|d z|$ satisfy

$$
\kappa \leq \lambda \leq C \kappa .
$$

The smallest such constant $C$ satisfies $h \leq C \leq k+\pi / 2$. For simply connected domains, this improves to $h \leq C \leq k$.

When $\Omega$ is a hyperbolic plane domain, we can fix one of the points $a, b, c$ to be the point at infinity and thus obtain a metric $\tilde{\kappa}(z)|d z|$. Evidently, $\tilde{\kappa} \leq \kappa$. Sugawa and Vuorinen studied the metric $\tilde{\kappa}(z)|d z|$ (as did Gardiner and Lakic) and proved that $\lambda \leq C \tilde{\kappa}$ with $k \leq C \leq 2 k+\pi / 2$ [SV05, Lemma 3.1]. Very recently, Betsakos Bet08, Theorem 1(c)] established that $\lambda \leq 8.27 \tilde{\kappa}$.

Among the metrics we consider, $\kappa(z)|d z|$ provides the best approximation for the hyperbolic metric in that it gives the largest lower bound. In addition, this metric has the best analytic properties being a continuous ultrahyperbolic metric having generalized Gaussian curvature at most -1 ; see Proposition 3.4. However, one is faced with the problem of determining the extremal points $a, b, c$. This can be difficult, even for elementary simply connected domains; see Section 4 . Moreover, even if one can locate the extremal points, one is then confronted with calculating or estimating $\lambda_{a b c}$. On the other hand, the metric $\alpha(z)|d z|$ is fairly simple to compute, but it gives the worst (i.e., smallest) lower bound and has the worst analytic properties. In fact, in general it is not even continuous!

As a useful compromise, we introduce the metric $\varepsilon(z)|d z|=\varepsilon_{\Omega}(z)|d z|$ defined for any region $\Omega \subset \hat{\mathbb{C}}$ and for $z \in \Omega \cap \mathbb{C}$ via

$$
\varepsilon(z)=\varepsilon_{\Omega}(z):=\sup _{a, b, c \in \Omega^{c}} \frac{|a-b|}{|z-a||z-b|} \cdot \frac{1}{k+|\log | z, a, b, c||} .
$$

Like $\alpha(z)|d z|$, the metric $\varepsilon(z)|d z|$ can be described geometrically, so one can determine the extremal points and then explicitly calculate the metric. This metric is continuous and provides a better lower bound than $\alpha(z)|d z|$. Another consequence of Theorem $\mathrm{A}$ is that $\varepsilon(z)|d z|$ is bilipschitz equivalent to the hyperbolic metric.

Corollary D. For any hyperbolic region $\Omega$ in $\hat{\mathbb{C}}$, the metrics $\varepsilon(z)|d z|$ and $\lambda(z)|d z|$ satisfy

$$
\varepsilon \leq \lambda \leq C \varepsilon
$$

The smallest such constant $C$ satisfies $k \leq C \leq k+\pi / 2$. For simply connected domains, the sharp constant is $C=k$. 
In this article we examine the three conformal metrics $\alpha(z)|d z|, \varepsilon(z)|d z|, \kappa(z)|d z|$ each of which is Möbius invariant and bilipschitz equivalent to the hyperbolic metric, with explicit absolute bilipschitz constants which are independent of the region $\Omega$. When $\Omega$ is a plane region (i.e., $\Omega \subset \mathbb{C}$ ) there are affine analogs $\tilde{\alpha}(z)|d z|, \tilde{\varepsilon}(z)|d z|$, $\tilde{\kappa}(z)|d z|$ which are affine invariant and are also bilipschitz equivalent to $\lambda(z)|d z|$.

This document is organized as follows: Section 2 contains preliminary information including definitions and terminology as well as elementary or well-known facts. In Section 3 we verify Möbius invariance and establish basic properties of the metrics and we study the geometry underlying $\gamma$. We exhibit numerous examples in Section 4, several of which illustrate the difficulty of determining the Gardiner-Lakic metrics. We conclude with Section 5 where we corroborate the results stated above. In fact, all of the inequalities displayed above in Theorem $\mathrm{A}$ and Corollaries $\mathrm{B}, \mathrm{C}$, D. as well as the Gardiner-Lakic and Sugawa-Vuorinen inequalities, are part of a more general framework involving all seven of these metrics; see Theorem 5.1 and $\$ 5 . \mathrm{B}$. In fact, these six metrics are also quasiinvariant with respect to holomorphic covering projections as revealed in Proposition 5.5.

\section{Preliminaries}

2.A. General information. Our notation is relatively standard. We work in the complex plane $\mathbb{C}$; stated results are valid for the Riemann sphere $\hat{\mathbb{C}}=\mathbb{C} \cup\{\infty\}$ in terms of local coordinates as the reader may verify. Everywhere $\Omega$ is a domain (i.e., an open connected set) and $\Omega^{c}, \partial \Omega$ denote the complement, boundary (respectively) of $\Omega$ with respect to $\hat{\mathbb{C}}$. The Euclidean line segment joining points $a, b \in \mathbb{C}$ is $[a, b]$, and $(a, b)=[a, b] \backslash\{a, b\}$. The Euclidean disk centered at the point $a \in \mathbb{C}$ of radius $r$ is denoted by $D(a ; r)$ and $\mathbb{D}:=D(0 ; 1)$ is the unit disk. We also let $\mathbb{H}$ denote the upper half-plane, $\mathbb{H}:=\{\Im(z)>0\}$, and we define

$$
\mathbb{C}^{*}:=\mathbb{C} \backslash\{0\}, \quad \mathbb{C}_{a b}:=\mathbb{C} \backslash\{a, b\}, \quad \hat{\mathbb{C}}_{a b}:=\hat{\mathbb{C}} \backslash\{a, b\}, \quad \hat{\mathbb{C}}_{a b c}:=\hat{\mathbb{C}} \backslash\{a, b, c\} ;
$$

the latter definitions are for distinct points $a, b$ in $\mathbb{C}$ or $a, b, c$ in $\hat{\mathbb{C}}$.

The quantity $\delta(z)=\delta_{\Omega}(z):=\operatorname{dist}(z, \partial \Omega)=\operatorname{dist}\left(z, \Omega^{c} \cap \mathbb{C}\right)$ is the Euclidean distance from $z \in \mathbb{C}$ to the boundary of $\Omega$, and $1 / \delta$ is the density for the so-called quasihyperbolic metric $|d z| / \delta(z)$ when $\Omega \subset \mathbb{C}$; see 2 .C We make frequent use of the notation

$$
D(z)=D_{\Omega}(z):=D(z ; \delta(z))=D\left(z ; \delta_{\Omega}(z)\right)
$$

for the maximal Euclidean disk in $\Omega$ centered at $z$.

As our notation suggests, we do not include $\Omega$ whenever the region is clear from context. Often, if there are two regions in consideration, say $\Omega$ and $\Omega^{\prime}$, we will use a prime $\left(^{\prime}\right)$ to indicate quantites associated with $\Omega^{\prime}$. For example, $\delta^{\prime}(z)=\delta_{\Omega^{\prime}}(z)$.

Recall that the circles $C$ on $\hat{\mathbb{C}}$, which have the property that two given points $a, b \in \hat{\mathbb{C}}$ are symmetric with respect to $C$, are called the circles of Apollonius with limit points $a, b$; see Ahl79, p. 85]. Of course, when one of $a$ or $b$ is the point at infinity, we obtain a family of concentric circles. However, when both $a, b \in \mathbb{C}$, these circles are given by the equations $|z-a|=t|z-b|$ with $t>0$; for $t=1$ we obtain the perpendicular bisector of $[a, b]$ (together with the point at infinity) and for $t>1(0<t<1$ respectively) the circle encloses $b$ ( $a$ respectively). 


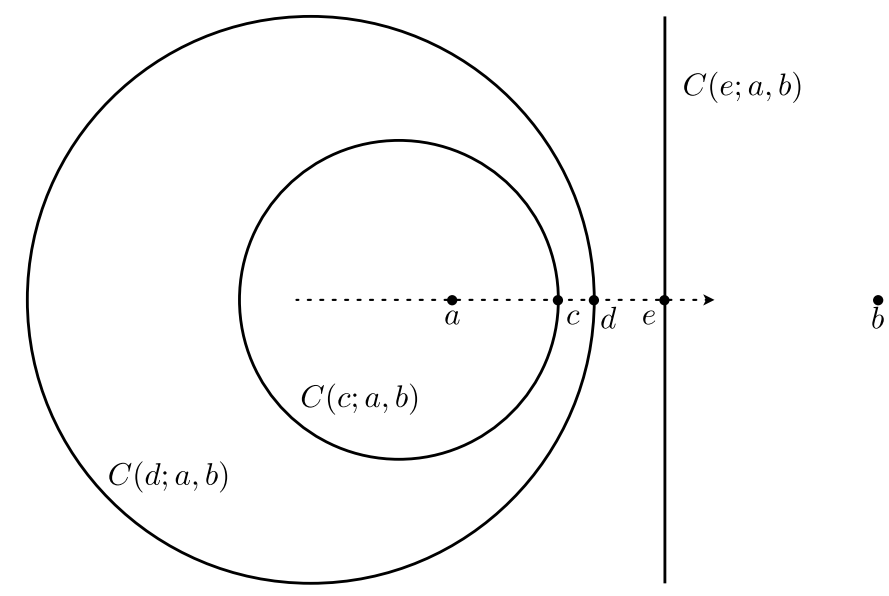

Figure 1. Apollonian circles with limit points $a, b$; here $e=(a+b) / 2$

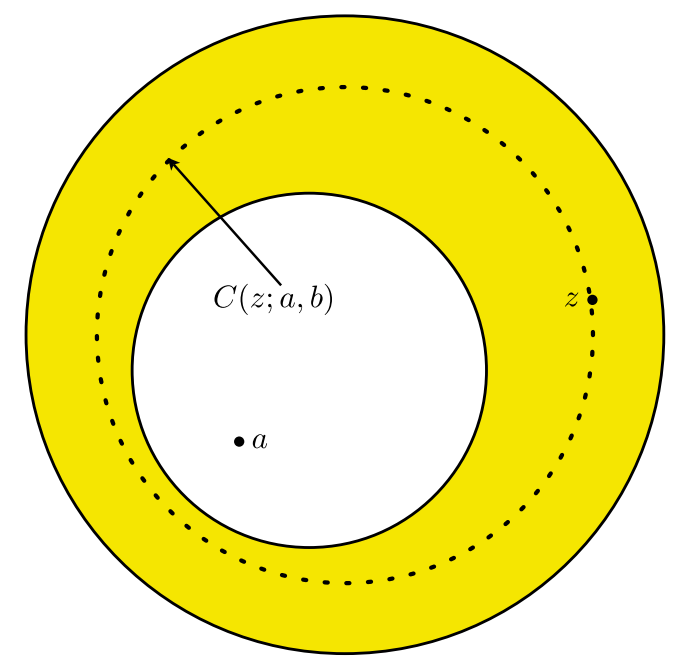

Figure 2. A Möbius annulus symmetric about $C(z ; a, b)$

For distinct points $z, a, b \in \hat{\mathbb{C}}$, we let $C(z ; a, b)$ denote the Apollonian circle through $z$ with limit points $a, b$ (see Figure 1). Thus when $a, b \in \mathbb{C}$,

$$
C(z ; a, b):=\left\{\zeta \in \hat{\mathbb{C}}:\left|\frac{\zeta-a}{\zeta-b}\right|=\left|\frac{z-a}{z-b}\right|\right\}=\{\zeta \in \hat{\mathbb{C}}:|\zeta, a, b, z|=1\} .
$$

Notice that for any $t>0$,

$$
A(z ; a, b, t):=\left\{\zeta \in \hat{\mathbb{C}}: e^{-t}<|\zeta, a, b, z|<e^{t}\right\}
$$

is a Möbius annulus (i.e., the Möbius image of a round Euclidean annulus) which is symmetric with respect to $C(z ; a, b)$ (see Figure 2). Moreover, the conformal modulus of $A(z ; a, b, t)$ is $\bmod A(z ; a, b, t)=2 t$. 
2.B. Conformal metrics. Recall that a conformal metric on a region $\Omega \subset \mathbb{C}$ has the form $\rho(z)|d z|$ where $\rho$ is some positive Borel function defined on $\Omega$ (with the property that the line element $\rho(z)|d z|$ integrates to an honest distance function); we call such a $\rho$ a metric-density. When $\Omega$ contains the point at infinity, we must use local coordinates and remember that we are dealing with a metric. Here we consider several such metrics.

When we have two conformal metrics, say $\rho(z)|d z|$ and $\sigma(z)|d z|$, defined on some $\Omega$, their ratio $\rho(z)|d z| / \sigma(z)|d z|$ is a well-defined positive function on $\Omega$. We write $\rho \leq C \sigma$ to indicate that this metric ratio is bounded above by $C$.

We remind the reader that whenever $f: \Omega \rightarrow \Omega^{\prime}$ is a (locally univalent) holomorphic map, every conformal metric $\sigma(w)|d w|$ on $\Omega^{\prime}$ determines the so-called pullback metric on $\Omega$ given by

$$
\rho(z)|d z|=f^{*}[\sigma(w)|d w|]:=\sigma(f(z))\left|f^{\prime}(z)\right||d z| .
$$

Sometimes we abuse notation and abbreviate this by writing $\rho=f^{*}[\sigma]$.

2.C. Hyperbolic, quasihyperbolic and Ferrand metrics. When $\Omega \subset \hat{\mathbb{C}}$ has at least three boundary points, usually dubbed a hyperbolic domain, there exists a unique metric $\lambda(z)|d z|=\lambda_{\Omega}(z)|d z|$ on $\Omega$ which enjoys the property that its pullback $p^{*}[\lambda(z)|d z|]$, with respect to any holomorphic universal covering projection $p: \mathbb{D} \rightarrow \Omega$, is the hyperbolic metric $\lambda_{\mathbb{D}}(\zeta)|d \zeta|=2\left(1-|\zeta|^{2}\right)^{-1}|d \zeta|$ on $\mathbb{D}$. In terms of such a covering $p$, the metric-density $\lambda=\lambda_{\Omega}$ of the Poincaré hyperbolic metric $\lambda_{\Omega}(z)|d z|$ can be determined from

$$
\lambda(z)=\lambda_{\Omega}(z)=\lambda_{\Omega}(p(\zeta)):=2\left(1-|\zeta|^{2}\right)^{-1}\left|p^{\prime}(\zeta)\right|^{-1} ;
$$

of course this is only valid at points $z=p(\zeta) \in \Omega \cap \mathbb{C}$. Yet another description is that $\lambda(z)|d z|$ is the maximal constant curvature -1 metric on $\Omega$.

Here is a perhaps surprising extension of Schwarz' Lemma. A proof can be modeled on the argument for [Min82, Theorem 1].

Fact 2.1. Let $\Omega, \Omega^{\prime}$ be hyperbolic regions. Assume $f$ is holomorphic in some neighborhood of $a \in \Omega$ and takes values in $\Omega^{\prime}$. Suppose that for all $z$ near $a$, $f^{*}\left[\lambda^{\prime}\right](z)=\lambda^{\prime}(f(z))\left|f^{\prime}(z)\right| \leq \lambda(z)$ with equality holding at $z=a$. Then $f$ extends to a holomorphic covering projection $f: \Omega \rightarrow \Omega^{\prime}$; in particular, $f^{*}\left[\lambda^{\prime}\right]=\lambda$.

We write $\lambda_{a b c}(z)|d z|$ for the hyperbolic metric in the thrice punctured sphere $\hat{\mathbb{C}}_{a b c}$, and $\lambda_{a b}(z)|d z|$ is an abbreviation for $\lambda_{a b \infty}(z)|d z|$, the hyperbolic metric in $\mathbb{C}_{a b}$. The 'standard' thrice punctured sphere is $\mathbb{C}_{01}$ and its hyperbolic metric has been extensively studied by numerous researchers including [Hem79, Min87, SV01, and [SV05]. Here we only mention the following information (which we require in the sequel).

Facts 2.2. (a) The metric-density $\lambda_{01}$ enjoys the symmetry properties

$$
\lambda_{01}(z)=\lambda_{01}(\bar{z})=\lambda_{01}(1-z)=\frac{1}{|z|^{2}} \lambda_{01}(1 / z) .
$$

(b) We have the lower bounds $\lambda_{01}(z) \geq \lambda_{01}(-|z|)$ and

$$
\lambda_{01}(z) \geq(|z|[k+|\log | z||])^{-1} \quad \text { with equality at } z \text { if and only if } z=-1 \text {. }
$$

(c) For any distinct $z, a, b, c \in \hat{\mathbb{C}}$,

$$
\lambda_{a b c}(z)=\frac{|a-b||b-c|}{|z-b|^{2}|a-c|} \lambda_{01}([z, a, b, c]) .
$$


(d) For fixed $\theta \in \mathbb{R}, r \mapsto r \lambda_{01}\left(r e^{i \theta}\right)$ is strictly increasing (decreasing) for $r \in(0,1)$ $(r>1$, respectively). Consequently,

$$
\frac{\partial}{\partial r}\left[r \lambda_{01}\right]=\lambda_{01}+r \frac{\partial \lambda_{01}}{\partial r}=\lambda_{01}+x \frac{\partial \lambda_{01}}{\partial x}+y \frac{\partial \lambda_{01}}{\partial y} \begin{cases}>0, & \forall r \in(0,1), \\ <0, & \forall r>1 .\end{cases}
$$

(e) For $z=x+i y \in \mathbb{D} \backslash \bar{D}(1 ; 1), \frac{\partial \lambda_{01}}{\partial x}>0$.

Parts (a) and (c) above are easily seen by making the appropriate Möbius change of variables. The lower bound in (b) is due to Hempel Hem79 and Jenkins Jen81; see also [Min87, Theorem 4]. The monotonicity property in (d) was established by Hempel [Hem79]; see also Min87, Theorem 3(iii)]. To confirm that (e) holds, notice that by $(\mathrm{d})$ we have

$$
\forall|z|<1: \quad \lambda_{01}+x \frac{\partial \lambda_{01}}{\partial x}+y \frac{\partial \lambda_{01}}{\partial y}>0
$$

and

$$
\forall|z-1|>1: \quad \lambda_{01}+(x-1) \frac{\partial \lambda_{01}}{\partial x}+y \frac{\partial \lambda_{01}}{\partial y}<0
$$

whence

$$
\forall|z|<1<|z-1|: \quad(x-1) \frac{\partial \lambda_{01}}{\partial x}<x \frac{\partial \lambda_{01}}{\partial x} .
$$

As seen from Fact 2.2(b), the constant $k$ is connected with sharp bounds in Landau's theorem. In fact, both of the constants $k$ and $h$ arise as special values of the hyperbolic metric-density $\lambda_{01}(z)$. Indeed,

$$
k=\frac{1}{\lambda_{01}(-1)} \quad \text { and } \quad h=\frac{2 / \sqrt{3}}{\lambda_{01}(\tau)}, \text { where } \tau=e^{\pi i / 3}=(1+i \sqrt{3}) / 2 .
$$

According to Fact 2.2(a), $\lambda_{01}(-1)=(1 / 4) \lambda_{01}(1 / 2)$. Also,

$$
\lambda_{01}(1 / 2)=\frac{16 \pi^{2}}{\Gamma^{4}(1 / 4)}=0.913893 \ldots \quad \text { and } \quad \lambda_{01}(\tau)=\frac{2^{2 / 3} \cdot 8 \pi^{3}}{3 \Gamma^{6}(1 / 3)}=0.355082 \ldots
$$

The elliptic modular function $J: \mathbb{H} \rightarrow \mathbb{C}_{01}$ satisfies $J(i)=1 / 2$ and $J(\tau)=\tau$; of course, $\lambda_{01}(J(\zeta))=\left[\operatorname{Im}(\zeta)\left|J^{\prime}(\zeta)\right|\right]^{-1}$. Thus, the first formula displayed above follows from Nehari's calculation [Neh75, (97), p. 329] of $\left|J^{\prime}(i)\right|$ and the second formula is a consequence of Carathéodory's computation Car60, p. 193] of $\left|J^{\prime}(\tau)\right|$. The decimal expressions are easily calculated using Mathematica (which also has the builtin ModularLambda function permitting a direct calculation of these values:-).

Our intuition tells us that if we 'move' points of the boundary closer, then the hyperbolic metric gets bigger. The following result clarifies this in the special case of punctured spheres.

Lemma 2.3. Let $D$ be a disk in $\hat{\mathbb{C}}$. Let $A$ be a discrete set of points in $\hat{\mathbb{C}} \backslash D$ with cardinality at least three. Let $S \subset A$. Put $\Omega=\hat{\mathbb{C}} \backslash\left[S^{*} \cup(A \backslash S)\right]$ where $S^{*}$ denotes the reflection of $S$ across $\partial D$. Then for all $z \in \bar{D} \cap \Omega, \lambda_{\widehat{\mathbb{C}} \backslash A}(z) \leq \lambda_{\Omega}(z)$.

Proof. The polarization of $\Omega$ across $\partial D$ is $P(\Omega)=\hat{\mathbb{C}} \backslash A$; see [Sol97].

The following monotonicity properties, first noticed by [SV01, Corollary 2.14], are straightforward special cases of the above. 
Facts 2.4. The quantity $\lambda_{a 1}(0)$, with $a=r e^{i \theta}$, possesses the following properties:

(a) For fixed $\theta \in[\pi / 2,3 \pi / 2], \lambda_{a 1}(0)$ is a decreasing function of $r \in(0, \infty)$.

(b) For fixed $\theta \in \mathbb{R}, \lambda_{a 1}(0)$ is a decreasing function of $r \in(0,1)$.

(c) For fixed $r>0, \lambda_{a 1}(0)$ is an increasing function of $\theta \in(0, \pi)$.

As alluded to above, for proper subdomains $\Omega \subsetneq \mathbb{C}$, the so-called quasihyperbolic metric is given by $|d z| / \delta(z)$. This metric has proven useful in many areas of geometric analysis. The quasihyperbolic metric in the punctured plane $\mathbb{C}^{*}$ is simply $|d z| /|z|$, which classically was called the logarithmic metric. Employing an auxiliary Möbius transformation, we define a Möbius invariant analog of this metric in the region $\hat{\mathbb{C}}_{a b}$ by

$$
\tau_{a b}(z):=\frac{|a-b|}{|z-a||z-b|},
$$

with the standard interpretation if one of $a$ or $b$ is the point at infinity (in which case $\tau_{a b}$ reduces to the quasihyperbolic metric on the appropriate punctured plane). That $\tau_{a b}(z)|d z|$ is Möbius invariant follows by a direct calculation. Alternatively, one can obtain this by realizing that

$$
\tau_{a b}(z)=\lim _{w \rightarrow z} \frac{|w, z, a, b|}{|w-z|} .
$$

The density for the Ferrand metric $\varphi_{\Omega}(z)|d z|$, introduced in Fer88, is given for $z \in \Omega \cap \mathbb{C}$ by

$$
\varphi(z)=\varphi_{\Omega}(z):=\sup _{a, b \in \Omega^{c}} \tau_{a b}(z) .
$$

In fact, there exist points $a, b \in \partial \Omega$ such that $\varphi(z)=\tau_{a b}(z)$. One way to see this is to notice that $\varphi(z)=\operatorname{diam}\left(B_{z}\right)$ where $B_{z}$ is the complement of the image of $\Omega$ under the inversion $\zeta \mapsto 1 /(\zeta-z)$; see [HIM08, Proposition 4.4]. The Ferrand metric is a Möbius invariant analog of the quasihyperbolic metric and enjoys the properties that

$$
\frac{1}{\delta} \leq \varphi \leq \frac{2}{\delta} \quad \text { and } \quad \lambda \leq \varphi .
$$

The leftmost inequality above is only valid for plane regions whereas the other two inequalities are always in force. We have $\varphi(z)=2 / \delta(z)$ if and only if there are a pair of diametrically opposite points in $\partial D(z) \cap \partial \Omega$. The rightmost inequality was established by Solynin Sol99a, Theorem 3] who also proved that equality holds at a single point if and only if the region is a disk in $\hat{\mathbb{C}}$ (in which case $\varphi(z)|d z|=\lambda(z)|d z|)$.

According to our notational conventions, we should define $\tilde{\varphi}:=1 / \delta$.

2.D. Beardon-Pommerenke and Gardiner-Lakic metrics. Beardon and Pommerenke [BP78] established an especially useful geometric estimate for the hyperbolic metric. They introduced the domain function

$$
\beta(z)=\beta_{\Omega}(z):=\inf _{a, c \in \Omega^{c}}|\log | \frac{z-a}{c-a}||
$$

where the infimum is restricted to the nearest boundary points $a \in \partial \Omega \cap \partial D(z)$ for $z$ (i.e., $\delta(z)=|z-a|)$; here $\Omega$ is assumed to be a plane domain. Clearly $\beta$ is affine invariant: the change of variables $w=a z+b$ maps $\Omega$ to another plane domain $\Omega^{\prime}$ 
and we easily see that $\beta^{\prime}(w)=\beta(z)$. For hyperbolic plane regions $\Omega$ we define the Beardon-Pommerenke metric $\tilde{\alpha}(z)|d z|=\tilde{\alpha}_{\Omega}(z)|d z|$ by

$$
\tilde{\alpha}(z)=\tilde{\alpha}_{\Omega}(z):=\frac{1}{\delta(z)} \frac{1}{k+\beta(z)} .
$$

It is evident that $\tilde{\alpha}(z)|d z|$ is invariant under similarities of $\mathbb{C}$.

The definition of $\beta$ can be motivated by examining the standard lower bound for the hyperbolic metric on a twice punctured plane; see Fact 2.2 (b) and also $3 . \mathrm{B}$. Beardon and Pommerenke BP78, Theorem 1] established the inequalities

$$
\forall z \in \Omega: \quad \tilde{\alpha}(z) \leq \lambda(z) \leq \frac{\pi / 2}{\delta(z) \beta(z)} .
$$

These inequalities, in conjunction with the classical estimate $\lambda \leq 2 / \delta$, immediately yield

$$
\forall z \in \Omega: \quad \tilde{\alpha}(z) \leq \lambda(z) \leq(2 k+\pi / 2) \tilde{\alpha}(z) .
$$

To obtain a Möbius invariant analog of $\beta$ which is valid for regions in $\hat{\mathbb{C}}$, we introduce

$$
\gamma(z)=\gamma_{\Omega}(z):=\inf _{a, b, c \in \Omega^{c}}|\log | z, a, b, c||
$$

where the infimum is restricted to those points $a, b \in \Omega^{c}$ which satisfy $\varphi(z)=\tau_{a b}(z)$. The related quantity $\tilde{\gamma}(z)$, defined for plane domains and obtained by fixing $b$ as the point at infinity, is of course $\tilde{\gamma}=\beta$.

As in the Introduction, we now have metrics $\alpha(z)|d z|$ and $\tilde{\alpha}(z)|d z|$, for regions in $\hat{\mathbb{C}}$ and $\mathbb{C}$ respectively, defined for $z \in \Omega \cap \mathbb{C}$ via

$$
\alpha(z)=\alpha_{\Omega}(z):=\frac{\varphi(z)}{k+\gamma(z)} \quad \text { and } \quad \tilde{\alpha}(z)=\tilde{\alpha}_{\Omega}(z):=\frac{\tilde{\varphi}(z)}{k+\tilde{\gamma}(z)}=\frac{1}{\delta(z)} \frac{1}{k+\beta(z)} .
$$

We readily find that these are Möbius and affine invariant respectively (as metrics).

Next, the Gardiner-Lakic metric $\kappa(z)|d z|=\kappa_{\Omega}(z)|d z|$ is defined for every hyperbolic region $\Omega$ in $\hat{\mathbb{C}}$ by

$$
\kappa(z)=\kappa_{\Omega}(z):=\sup _{a, b, c \in \Omega^{c}} \lambda_{a b c}(z)
$$

where $z \in \Omega \cap \mathbb{C}$. They also studied the related metrics obtained by taking $a$ to be the point at infinity (for a plane region) or by taking $a$ to be a boundary point nearest to $z$. For hyperbolic plane domains, the metric $\tilde{\kappa}(z)|d z|=\tilde{\kappa}_{\Omega}(z)|d z|$ is obtained by fixing one of the points in the above supremum to be the point at infinity. Thus, for $z \in \Omega \subset \mathbb{C}$ we let

$$
\tilde{\kappa}(z)=\tilde{\kappa}_{\Omega}(z):=\sup _{a, b \in \Omega^{c}} \lambda_{a b}(z) .
$$

For the record, we also define $\tilde{\varepsilon}(z)|d z|=\tilde{\varepsilon}_{\Omega}(z)|d z|$ for hyperbolic plane regions $\Omega$ via

$$
\tilde{\varepsilon}(z)=\tilde{\varepsilon}_{\Omega}(z):=\sup _{a, c \in \Omega^{c}} \frac{1}{|z-a|} \cdot \frac{1}{k+|\log | z-a|/| c-a||} ;
$$

here $z \in \Omega \subset \mathbb{C}$, and $a, c \in \Omega^{c} \cap \mathbb{C}$ are distinct. 


\section{BASIC PROPERTIES}

That each of the metrics $\alpha(z)|d z|, \varepsilon(z)|d z|, \kappa(z)|d z|$ enjoys the domain monotonicity property that larger domains have smaller metrics is a straightforward consequence of their definitions. In this section we discuss invariance properties of these metrics, describe a geometric interpretation for $\gamma$ (and $\beta$ ), and establish some elementary properties concerning the location of extremal punctures as well as continuity (or lack thereof) for the metrics.

3.A. Möbius invariance. We demonstrate that $\alpha(z)|d z|, \varepsilon(z)|d z|$ and $\kappa(z)|d z|$ are all Möbius invariant. The related metrics, $\tilde{\alpha}|d z|, \tilde{\varepsilon}|d z|, \tilde{\kappa}|d z|$ are affine invariant.

This is clear for $\alpha(z)|d z|$, because both $\varphi(z)|d z|$ and $\gamma(z)$ are Möbius invariant. Similar reasoning holds for $\varepsilon(z)|d z|$ : both $\tau_{a b}(z)|d z|$ and $|\log | z, a, b, c||$ are Möbius invariant. Alternatively, we can use the fact that $\varepsilon(z)$ is found by maximizing $\tau_{a b}(z) /[k+\gamma(z ; a, b)]$ as explained in $§ 3 . \mathrm{B}$. It remains to consider $\kappa(z)|d z|$.

Suppose $T$ is a Möbius transformation and $\Omega^{\prime}=T(\Omega)$. We want to show that

$$
T^{*}\left[\kappa^{\prime}(w)|d w|\right]=\kappa(z)|d z| .
$$

Note that $a, b, c \in \Omega^{c}$ if and only if their images $a^{\prime}=T(a), b^{\prime}=T(b), c^{\prime}=T(c)$ lie in $\hat{\mathbb{C}} \backslash \Omega^{\prime}$. Also, $T^{*}\left[\lambda_{a^{\prime} b^{\prime} c^{\prime}}(w)|d w|\right]=\lambda_{a b c}(z)|d z|$. Let $a, b, c \in \Omega^{c}$ be distinct. Then

$$
\lambda_{a^{\prime} b^{\prime} c^{\prime}}(w)|d w| \leq \kappa^{\prime}(w)|d w|
$$

SO

$$
\lambda_{a b c}(z)|d z|=T^{*}\left[\lambda_{a^{\prime} b^{\prime} c^{\prime}}(w)|d w|\right] \leq T^{*}\left[\kappa^{\prime}(w)|d w|\right] .
$$

Therefore, $\kappa(z)|d z| \leq T^{*}\left[\kappa^{\prime}(w)|d w|\right]$. Applying the same argument to $T^{-1}$ yields

$$
\kappa^{\prime}(w)|d w| \leq\left(T^{-1}\right)^{*}[\kappa(z)|d z|]
$$

from which we deduce the opposite inequality

$$
T^{*}\left[\kappa^{\prime}(w)|d w|\right] \leq T^{*}\left(\left(T^{-1}\right)^{*}[\kappa(z)|d z|]\right)=\kappa(z)|d z|
$$

Similarly, one can show that all three metrics are invariant under reflection in a circle or line. More generally, they are invariant under any conjugate Möbius transformation.

3.B. The geometry of $\gamma$ and $\tilde{\gamma}$. Throughout this subsection $\Omega$ is a fixed hyperbolic region in $\hat{\mathbb{C}}$ or in $\mathbb{C}$ as indicated by context. We present a geometric interpretation for the quantities $\gamma(z)$ and $\tilde{\gamma}(z)$. This in turn yields an algorithm for computing $\alpha(z)$ and $\tilde{\alpha}(z)$. Similar ideas apply for the metric-densities $\varepsilon(z)$ and $\tilde{\varepsilon}(z)$.

We start by defining quantities $\gamma(z ; a, b)$, and $\tilde{\gamma}(z ; a)$, for distinct points $a, b \in \Omega^{c}$, and $a \in \Omega^{c} \cap \mathbb{C}$ respectively, as follows:

$\gamma(z ; a, b):=\inf _{c \in \Omega^{c} \backslash\{a, b\}}|\log | z, a, b, c|| \quad$ and $\quad \tilde{\gamma}(z ; a):=\inf _{c \in \Omega^{c} \backslash\{a, \infty\}}|\log | z-a|/| c-a||$.

Note that $|z-a| /|c-a|=|z, a, \infty, c|$, so $\tilde{\gamma}(z ; a)=\gamma(z ; a, \infty)$. Evidently,

$$
\gamma(z)=\inf _{a, b} \gamma(z ; a, b) \text { and } \quad \tilde{\gamma}(z)=\inf _{a} \tilde{\gamma}(z ; a)
$$

where the infima are restricted to those points $a, b \in \Omega^{c}$ which satisfy $\varphi(z)=\tau_{a b}(z)$ and $\delta(z)=|z-a|$ respectively.

We note that for distinct $a, b \in \Omega^{c}$ and $z \in \Omega$, there always exists a point $c \in \Omega^{c} \backslash\{a, b\}$ with $\gamma(z ; a, b)=|\log | z, a, b, c||$. Indeed, from any sequence $\left(c_{n}\right)_{n=1}^{\infty}$ in 
$\Omega^{c} \backslash\{a, b\}$ with $|\log | z, a, b, c_{n}|| \rightarrow \gamma(z ; a, b)$, we may extract a convergent subsequence and so assume that $c_{n} \rightarrow c \in \Omega^{c}$. Since $\gamma(z ; a, b)<\infty$, we have $c \in \Omega^{c} \backslash\{a, b\}$ as desired.

In our analysis of the metrics $\varepsilon(z)|d z|$ and $\tilde{\varepsilon}(z)|d z|$, we tacitly use the fact that these can be calculated via

$$
\varepsilon(z)=\sup _{a, b \in \Omega^{c}} \frac{\tau_{a b}(z)}{k+\gamma(z ; a, b)} \quad \text { and } \quad \tilde{\varepsilon}(z)=\sup _{a \in \Omega^{c}} \frac{1}{|z-a|[k+\tilde{\gamma}(z ; a)]} .
$$

Note that in the definitions of $\varepsilon$ and $\tilde{\varepsilon}$, there is no restriction on the points $a, b, c \in$ $\Omega^{c}$.

We discuss $\gamma(z ; a, b)$, but similar comments, appropriately modified, apply to $\tilde{\gamma}(z ; a)$.

Choose a point $c \in \Omega^{c} \backslash\{a, b\}$ with $\gamma(z ; a, b)=|\log | z, a, b, c||$. Let $C=C(z ; a, b)$ be the Apollonian circle through $z$ with limit points $a, b$; see the end of $2 . \mathrm{A}$. We have $\gamma(z ; a, b)=0$ if and only if $C \cap \partial \Omega \neq \emptyset$. Suppose $C \subset \Omega$, so $\gamma=\gamma(z ; a, b)>0$. Let

$$
A=A(z ; a, b, \gamma)=\left\{\zeta \in \hat{\mathbb{C}}: e^{-\gamma}<|z, a, b, \zeta|<e^{\gamma}\right\}
$$

Of course $2 \gamma=\bmod (A)$ is the conformal modulus of $A$. We claim that $A$ is the maximal Möbius annulus which is contained in $\Omega$ and is symmetric with respect to $C$. This is because on the one hand, the extremal point $c$ lies on $\partial A$, so $\partial A \cap \partial \Omega \neq \emptyset$. On the other hand, from the definition of $\gamma(z ; a, b)$ we must have $A \subset \Omega$.

Notice that when $\gamma(z ; a, b)>0$, any extremal point $c \in \Omega^{c}$ (with $\gamma(z ; a, b)=$ $|\log | z, a, b, c||)$ must lie on $\partial \Omega$ (because then $c \in \partial A(z ; a, b, \gamma(z ; a, b)))$. This is not necessarily true when $\gamma(z ; a, b)=0$; nonetheless, even in this case we can still locate a point $d \in \partial \Omega$ with $\gamma(z ; a, b)=|\log | z, a, b, d||$ (simply by taking any $d \in \partial \Omega \cap C(z ; a, b, \gamma(z ; a, b)))$.

Thus, $2 \gamma(z ; a, b)$ is the conformal modulus of the maximal Möbius annulus that is contained in $\Omega$ and symmetric with respect to $C(z ; a, b)$. It follows that $\gamma(z)$ is the minimum of these numbers, where we only consider points $a, b \in \partial \Omega$ with $\tau_{a b}(z)=\varphi(z)$. We now see how to calculate the metric density $\alpha(z)$ (and likewise $\tilde{\alpha}(z))$ : For each pair of boundary points $a, b$ with $\varphi(z)=\tau_{a b}(z)$, we determine the largest Möbius annulus in $\Omega$ which is symmetric about $C(z ; a, b)($ so $\gamma(z ; a, b)$ is half its conformal modulus); then $\gamma(z)$ is the smallest of these maximal moduli.

There is one final point to emphasize here. The quantity $\gamma(z ; a, b)$ (and likewise $\tilde{\gamma}(z ; a)=\gamma(z ; a, \infty))$ is continuous in each of the parameters $z \in \Omega$ and $a, b \in \Omega^{c}$ (i.e., as functions of $\left.(z, a, b) \in \Omega \times \Omega^{c} \times \Omega^{c}\right)$. On the other hand, neither $\gamma(z)$ nor $\tilde{\gamma}(z)=\beta(z)$ are continuous! Indeed, for the punctured unit disk $\mathbb{D}^{*}$ we have $\tilde{\gamma}(z)=|\log | z||$ for $0<|z|<1 / 2$ whereas $\tilde{\gamma}(z)=0$ for $1 / 2 \leq|z|<1$.

We conclude this subsection with two examples showing first that $\gamma$ need not be continuous and second that $\gamma \leq \tilde{\gamma}$ need not hold.

For an example with $\gamma$ not continuous, let $\Omega=\mathbb{C} \backslash(\{$ iy : $|y| \geq 1\} \cup\{-3 / 4,3 / 2\})$. It is easy to check that $\gamma(0)=0$. Next, we examine $\gamma(z)$ for $z=x<0$ with $z$ near 0 . It is not difficult to check that for such $z$,

$$
\varphi(z)=\max \left\{\frac{9 / 4}{|z-3 / 2||z+3 / 4|}, \frac{\sqrt{13} / 2}{|z-3 / 2||z-i|}, \frac{5 / 4}{|z+3 / 4||z-i|}, \frac{2}{|z-i||z+i|}\right\}
$$


For $z=x<0$ sufficiently close to 0 we find that $\varphi(z)=\tau_{-3 / 4,3 / 2}(z)=$ $9 /(4|z-3 / 2||z+3 / 4|)$ and $-3 / 4,3 / 2$ are the only boundary points with this property. It is now evident that

$$
\lim _{z=x \nearrow 0} \gamma(z)=\lim _{z=x \nearrow 0}|\log | z,-3 / 4,3 / 2, i||=\log \frac{5}{\sqrt{13}} \neq 0 .
$$

An example where $\gamma(z)>\tilde{\gamma}(z)$ is provided by $\Omega=\mathbb{C} \backslash((-\infty,-1] \cup\{-i, t i\})$ where $1<t \leq 1+\sqrt{2}$. Clearly, $\tilde{\gamma}(0)=0$. Also, $\varphi(0)=1+1 / t$ which is attained by using the boundary points $-i, t i$. Evidently, $\gamma(0)=|\log | 0, t i,-i,-1||=$ $(1 / 2) \log \left(2 t^{2} /\left(1+t^{2}\right)\right)>0$.

3.C. Extremal punctures. The metric-densities $\alpha, \tilde{\alpha}, \varepsilon, \tilde{\varepsilon}, \kappa, \tilde{\kappa}$ are all defined as certain extreme values taken over points varying in $\Omega^{c}$. Because $\Omega^{c}$ is a closed subset of the compact space $\hat{\mathbb{C}}$, it is easy to see that there always exist extremal points. This is clear for the metrics $\alpha(z)|d z|$ and $\tilde{\alpha}(z)|d z|$, and not difficult to corroborate for the other metrics as well.

Here we investigate the question as to whether or not these extremal points lie on $\partial \Omega$. We saw in $\S 3$. B that $\gamma(z ; a, b)$ and $\tilde{\gamma}(z ; a)$ can always be calculated by looking at boundary points (although when one of these is zero, there may also be extremal points in $\hat{\mathbb{C}} \backslash \bar{\Omega})$. The actual definitions of $\alpha$ and $\tilde{\alpha}$ require that we look only at special boundary points. Thus it remains to consider $\varepsilon, \tilde{\varepsilon}, \kappa$, and $\tilde{\kappa}$.

Proposition 3.1. Let $\Omega \subset \hat{\mathbb{C}}$ be a hyperbolic region. For each $z \in \Omega$, there exist points $a, b, c \in \partial \Omega$ with $\varepsilon(z)=|a-b| /[|z-a||z-b|(k+|\log | z, a, b, c||)]$. Moreover, if $a, b, c \in \Omega^{c}$ have this property, then $a, b \in \partial \Omega$ (and $c \in \partial \Omega$ when $\gamma(z ; a, b)>0$ but not necessarily if $\gamma(z ; a, b)=0)$.

Proof. Fix $z \in \Omega$. By Möbius invariance, we may assume $z \in \mathbb{C}$. We begin by confirming that there are points $a, b, c \in \Omega^{c}$ such that $\varepsilon(z)=|a-b| /$ $[|z-a||z-b|(k+|\log | z, a, b, c||)]$. Select $a_{n}, b_{n} \in \Omega^{c}$ with $\tau_{a_{n} b_{n}}(z) /\left[k+\gamma\left(z ; a_{n}, b_{n}\right)\right] \rightarrow$ $\varepsilon(z)$. Extracting convergent subsequencs permits us to assume that $\left(a_{n}\right)$ and $\left(b_{n}\right)$ converge to points $a, b \in \Omega^{c}$. Since $\varepsilon(z)>0$, $\lim _{n \rightarrow \infty} \tau_{a_{n} b_{n}}(z)>0$, and therefore $a \neq b$. Now we choose $c \in \Omega^{c} \backslash\{a, b\}$ with $\gamma(z ; a, b)=|\log | z, a, b, c||$. This yields

$$
\varepsilon(z)=\lim _{n \rightarrow \infty} \frac{\tau_{a_{n} b_{n}}(z)}{k+\gamma\left(z ; a_{n}, b_{n}\right)}=\frac{\tau_{a b}(z)}{k+\gamma(z ; a, b)}=\frac{|a-b|}{|z-a||z-b|} \cdot \frac{1}{k+|\log | z, a, b, c||}
$$

as asserted.

Now we verify that the extremal points must be boundary points. Let $a, b, c \in \Omega^{c}$. Suppose $a \notin \partial \Omega$. We find a nearby point $d \in \Omega^{c}$ which provides a better (i.e., larger) potential value for $\varepsilon(z)$. Using Möbius invariance, we may assume that $b$ is the point at infinity. Then $z \in \mathbb{C}$ and there is some Euclidean disk $D=D(a ; r) \subset \Omega^{c}$. We exhibit such a point $d$ in $D \cap D(z ;|z-a|)$.

Let $C=C(a ; z, c)$ be the Apollonian circle through $a$ with limit points $z, c$. Suppose that $a \notin(z, c)$. Then the circle $C$ cuts inside the disk $D(z ;|z-a|)$ at the point $a$, so we may select a point $d \in C \cap D \cap D(z ;|z-a|)$. Then $|z-d|<|z-a|$, $d \in \Omega^{c}$ and $|z-d| /|c-d|=|z-a| /|c-a|$, so $a$ cannot be an extremal point for $\varepsilon(z)$.

Now we examine the situation when $a \in(z, c)$. In both cases we choose $d \in$ $(z, a) \cap D$, so $|z-d|<|z-a|$ and $d \in \Omega^{c}$. If $|z-a| /|c-a|>1$, then we can choose such a $d$ with $|z-a| /|c-a|>|z-d| /|c-d|>1$ and again we see that $a$ cannot be an extremal point for $\varepsilon(z)$. We are left with the case when $|z-a| /|c-a| \leq 1$. 
To simplify calculations, we now make an affine change of variables letting us assume that $z=0$ and $c=1$. Since $k>2$, an easy calculus exercise reveals that $f(x)=x\left[k+\log \left(x^{-1}-1\right)\right]$ is strictly increasing for $0<x \leq 1 / 2$. In particular, we can find $d \in(0, a) \cap D$ with $f(d)<f(a)$. Again, $a$ cannot be an extremal point for $\varepsilon(z)$.

Thus the only way $a, b \in \Omega^{c}$ can be extremal points for $\varepsilon(z)$ is if $a, b \in \partial \Omega$. The assertions regarding $c$ follow from the remarks near the end of $3 . \mathrm{B}$.

Notice that the above proof also applies to the metric $\tilde{\varepsilon}(z)|d z|$ : For each $z \in \Omega \subset$ $\mathbb{C}$, there exist $a, c \in \partial \Omega$ with $\tilde{\varepsilon}(z)=[|z-a|(k+|\log | z-a|/| c-a||)]^{-1}$. Moreover, if $a, c \in \Omega^{c}$ have this property, then $a \in \partial \Omega$ (but $c$ is not necessarily a boundary point).

Sugawa and Vuorinen confirmed that the extremal points for $\tilde{\kappa}(z)|d z|$ must be boundary points; see [SV05, p. 892]. Similar reasoning provides the last assertion in the following; for the reader's convenience, we indicate a proof.

Proposition 3.2. Let $\Omega \subset \hat{\mathbb{C}}$ be a hyperbolic region. For each $z \in \Omega$, there exist points $a, b, c \in \partial \Omega$ with $\kappa(z)=\lambda_{a b c}(z)$. Moreover, any $a, b, c \in \Omega^{c}$ which have this property must lie on $\partial \Omega$.

Proof. We first show that for each $z \in \Omega$ there exist $a, b, c \in \Omega^{c}$ with $\kappa(z)=\lambda_{a b c}(z)$. We need a result noted by Heins Hei62. Let $\mathcal{K}=\left\{\sigma_{\alpha}(z)|d z|: \alpha \in A\right\}$ denote the family of all conformal metrics with constant curvature -1 on $\Omega$. Then $\mathcal{K}$ is a normal family in the sense that any sequence $\left(\sigma_{n}(z)|d z|\right)_{n=1}^{\infty}$ in $\mathcal{K}$ contains a subsequence that converges locally uniformly in $\Omega$ to a metric $\sigma(z)|d z|$ and either $\sigma \equiv 0$ or else $\sigma(z)|d z| \in \mathcal{K}$.

Fix $z_{0} \in \Omega$. Choose $a_{n}, b_{n}, c_{n} \in \Omega^{c}$ so that $\lambda_{a_{n} b_{n} c_{n}}\left(z_{0}\right) \rightarrow \kappa\left(z_{0}\right)>0$. Because $\Omega^{c}$ is compact, we may assume that $a_{n} \rightarrow a, b_{n} \rightarrow b$ and $c_{n} \rightarrow c$. Also, since $\mathcal{K}$ is a normal family, we may assume that $\left(\lambda_{a_{n} b_{n} c_{n}}(z)\right)_{n=1}^{\infty}$ converges locally uniformly in $\Omega$. Since $\kappa\left(z_{0}\right)>0$, the limit metric belongs to $\mathcal{K}$. This implies that the points $a, b$ and $c$ are distinct and the limit is $\lambda_{a b c}(z)$. Consequently, $\kappa\left(z_{0}\right)=\lambda_{a b c}\left(z_{0}\right)$.

Alternatively, we could use the fact that if $f_{a b c}: \mathbb{D} \rightarrow \hat{\mathbb{C}}_{a b c}$ is the covering of $\mathbb{D}$ onto $\hat{\mathbb{C}}_{a b c}$ with $f(0)=z_{0}$ and $f^{\prime}(0)>0$, then $\mathcal{F}=\left\{f_{a b c}: a, b, c \in \Omega^{c}\right\}$ is a normal family. Also, if $a_{n} \rightarrow a, b_{n} \rightarrow b$ and $c_{n} \rightarrow c$, then $\left(f_{a_{n} b_{n} c_{n}}\right)$ converges to $f_{a b c}$ if the three points $a, b, c$ are distinct; otherwise it converges locally uniformly to $z_{0}$ (cf. Hej74).

It remains to confirm that extremal points must be boundary points. Fix $z \in \Omega$ and let $a, b, c \in \Omega^{c}$. Suppose $a \notin \partial \Omega$. We find a nearby point $d \in \Omega^{c}$ which provides a better (i.e., larger) potential value for $\kappa(z)$. Using Möbius invariance and symmetry, we may assume that $z, a, b, c$ are $0, r e^{i \theta}, 1, \infty$ respectively with $\theta \in[0, \pi]$.

First, suppose $\theta=\pi$; so $a=-r$. Select $s \in(0, r)$ so that $d=-s \in \Omega^{c}$. The monotonicity property Fact 2.4 (a) tells us that $\lambda_{d 1}(0)>\lambda_{a 1}(0)$. Now assume $\theta \in[0, \pi)$. Select $\vartheta \in(\theta, \pi)$ with $d=r e^{i \vartheta} \in \Omega^{c}$. Then Fact 2.4(c) tells us that $\lambda_{d 1}(z)>\lambda_{a 1}(z)$.

3.D. Analytic properties. At the end of $33 . \mathrm{B}$ we discussed the lack of continuity of $\gamma$ and $\tilde{\gamma}$. It follows that, in general, the metrics $\alpha(z)|d z|$ and $\tilde{\alpha}(z)|d z|$ are not continuous. However, the other four metrics are continuous. First we confirm this for $\varepsilon(z)|d z|$ and $\tilde{\varepsilon}(z)|d z|$.

Proposition 3.3. The metrics $\varepsilon(z)|d z|$ and $\tilde{\varepsilon}(z)|d z|$ are continuous. 
Proof. Fix $z_{0} \in \Omega \cap \mathbb{C}$ and select distinct $a, b \in \partial \Omega$ with $\varepsilon\left(z_{0}\right)=\tau_{a b}\left(z_{0}\right) /\left[k+\gamma_{a b}\left(z_{0}\right)\right]$. For any $z \in \Omega \cap \mathbb{C}, \varepsilon(z) \geq \tau_{a b}(z) /[k+\gamma(z ; a, b)]$. Since $\tau_{a b}$ and $\gamma(\cdot ; a, b)$ are continuous, it follows that $\liminf _{z \rightarrow z_{0}} \varepsilon(z) \geq \varepsilon\left(z_{0}\right)$. It remains to check the reverse inequality.

Let $\left(z_{n}\right)_{n=1}^{\infty}$ be any sequence in $\Omega \cap \mathbb{C}$ converging to $z_{0}$. Choose distinct $a_{n}, b_{n} \in$ $\partial \Omega$ with $\varepsilon\left(z_{n}\right)=\tau_{a_{n} b_{n}}\left(z_{n}\right) /\left[k+\gamma\left(z_{n} ; a_{n}, b_{n}\right)\right]$. Assume that $a_{n} \rightarrow a_{0}$ and $b_{n} \rightarrow b_{0}$. Then $a_{0} \neq b_{0}$, for otherwise we would get $\tau_{a_{n} b_{n}}\left(z_{n}\right) \rightarrow 0$ which would give $\varepsilon\left(z_{n}\right) \rightarrow 0$ contradicting $\liminf \operatorname{in}_{n \rightarrow \infty} \varepsilon\left(z_{n}\right) \geq \varepsilon\left(z_{0}\right)>0$.

Now, since $\tau_{a b}(z)$ and $\gamma(z ; a, b)$ are continuous in each of $z, a, b$, we see that

$$
\lim _{n \rightarrow \infty} \frac{\tau_{a_{n} b_{n}}\left(z_{n}\right)}{k+\gamma\left(z_{n} ; a_{n}, b_{n}\right)}=\frac{\tau_{a_{0} b_{0}}\left(z_{0}\right)}{k+\gamma\left(z_{0} ; a_{0}, b_{0}\right)} \leq \varepsilon\left(z_{0}\right)
$$

The above calculations confirm that $\limsup _{z \rightarrow z_{0}} \varepsilon(z) \leq \varepsilon\left(z_{0}\right)$ as desired.

In addition to being continuous, we can also provide curvature estimates for the metrics $\kappa(z)|d z|$ and $\tilde{\kappa}(z)|d z|$. Following Ahlfors [Ahl73, p. 13], a metric $\rho(z)|d z|$ is said to be ultrahyperbolic in $\Omega$ provided $\rho: \Omega \rightarrow[0,+\infty)$ is upper semicontinuous and at each point $a \in \Omega$ with $\rho(a)>0$, there exists a "supporting metric" $\sigma(z)|d z|$ which is defined and of class $\mathcal{C}^{2}$ in a neighborhood $U$ of $a$, has Gaussian curvature at most -1 , and satisfies $\rho \geq \sigma$ in $U$ with equality holding at the point $a$. An easy comparison lemma for Gaussian curvature (cf. HIM08, Lemma 2.12]) reveals that any ultrahyperbolic metric has generalized curvatures at most -1 .

Proposition 3.4. Let $\Omega \subset \hat{\mathbb{C}}$ be a hyperbolic region. Then $\kappa(z)|d z|$ is a continuous ultrahyperbolic metric on $\Omega$.

Proof. We first verify that $\kappa$ is a continuous function on $\Omega$. Fix $z_{0} \in \Omega$. We start by showing that

$$
\kappa\left(z_{0}\right) \leq \liminf _{z \rightarrow z_{0}} \kappa(z) .
$$

Let $\left(z_{n}\right)_{n=1}^{\infty}$ be a sequence of points in $\Omega$ converging to $z_{0}$ with $\kappa\left(z_{n}\right) \rightarrow$ $\liminf _{z \rightarrow z_{0}} \kappa(z)$. Choose $a, b, c \in \Omega^{c}$ with $\kappa\left(z_{0}\right)=\lambda_{a b c}\left(z_{0}\right)$. Then for all $n \in \mathbb{N}$, $\lambda_{a b c}\left(z_{n}\right) \leq \kappa\left(z_{n}\right)$, so

$$
\kappa\left(z_{0}\right)=\lambda_{a b c}\left(z_{0}\right)=\lim _{n \rightarrow \infty} \lambda_{a b c}\left(z_{n}\right) \leq \liminf _{n \rightarrow \infty} \kappa\left(z_{n}\right)=\liminf _{z \rightarrow z_{0}} \kappa(z) .
$$

This establishes lower semicontinuity. Now we prove upper semicontinuity. Let $\left(z_{n}\right)_{n=1}^{\infty}$ be a sequence of points in $\Omega$ converging to $z_{0}$ with $\kappa\left(z_{n}\right) \rightarrow \lim \sup _{z \rightarrow z_{0}} \kappa(z)$. For each $n \in \mathbb{N}$, choose $a_{n}, b_{n} c_{n} \in \Omega^{c}$ with $\kappa\left(z_{n}\right)=\lambda_{a_{n} b_{n} c_{n}}\left(z_{n}\right)$. We may assume that $a_{n}, b_{n}$, and $c_{n}$ converge to points $a, b, c \in \Omega^{c}$. A standard result (see Hej74] or Hei62] ) asserts that $\lambda_{a_{n} b_{n} c_{n}}(z) \rightarrow \lambda_{a b c}(z)$ locally uniformly in $\Omega$ provided $a, b$, and $c$ are all distinct. If they were not distinct, then the local uniform limit would be identically 0 , but the limit is positive because $\liminf _{z \rightarrow z_{0}} \kappa\left(z_{0}\right) \geq \kappa\left(z_{0}\right)>0$, so $a$, $b$, and $c$ must be distinct. Since $z_{n} \rightarrow z_{0}$, we deduce that $\lambda_{a_{n} b_{n} c_{n}}\left(z_{n}\right) \rightarrow \lambda_{a b c}\left(z_{0}\right)$. Thus,

$$
\lim _{n \rightarrow \infty} \kappa\left(z_{n}\right)=\lim _{n \rightarrow \infty} \lambda_{a_{n} b_{n} c_{n}}\left(z_{n}\right)=\lambda_{a b c}\left(z_{0}\right) .
$$

As $a, b, c \in \Omega^{c}$, we now obtain

$$
\kappa\left(z_{0}\right) \leq \liminf _{z \rightarrow z_{0}} \kappa(z) \leq \limsup _{z \rightarrow z_{0}} \kappa(z)=\lambda_{a b c}\left(z_{0}\right) \leq \kappa\left(z_{0}\right)
$$

which establishes continuity at $z_{0}$. 
Next, we demonstrate that $\kappa(z)|d z|$ has a supporting metric at each point of $\Omega$. Fix $z_{0} \in \Omega$. Select distinct $a, b, c \in \Omega^{c}$ with $\kappa\left(z_{0}\right)=\lambda_{a b c}\left(z_{0}\right)$. Then for all $z \in \Omega$, $\lambda_{a b c}(z) \leq \kappa(z)$ with equality at $z_{0}$. Since $\lambda_{a b c}(z)|d z|$ has constant curvature -1 , it is a supporting metric for $\kappa(z)|d z|$ at $z_{0}$. Thus $\kappa(z)|d z|$ is ultrahyperbolic and has generalized curvature at most -1 .

\section{EXAMPLES}

Here we mention a few special examples where one can actually calculate the hyperbolic, Ferrand, Gardiner-Lakic, Beardon-Pommerenke, and other related metrics. The reader should note that we also obtain information about all Möbius images of these special regions.

It is easy to see that in any simply connected region, $\gamma \equiv 0$, and thus

$$
\varepsilon=\alpha=\frac{\varphi}{k} \quad \text { and } \quad \tilde{\varepsilon}=\tilde{\alpha}=\frac{\tilde{\varphi}}{k}=\frac{1}{k \delta} .
$$

(In fact, by Lemma 5.3 we see that the above equalities hold at a given point $z$ precisely when $\gamma(z)=0$ or $\beta(z)=0$.) In particular, this means that for simply connected regions we are only interested in calculating $\kappa$ and $\tilde{\kappa}$. Also, the above, in conjunction with the 'classical' inequalities $\lambda \leq \varphi \leq 2 / \delta$ together with the basic inequalities from Theorem [5.1, yields

$$
\frac{\varphi}{k}=\alpha=\varepsilon \leq \lambda \leq k \alpha=\varphi \quad \text { and } \quad \frac{1}{k \delta}=\tilde{\alpha}=\tilde{\varepsilon} \leq \lambda \leq 2 k \tilde{\alpha}=\frac{2}{\delta} .
$$

On the other hand, the classical inequalities

$$
\frac{1}{2 \delta} \leq \frac{\varphi}{2} \leq \lambda \leq \varphi \leq \frac{2}{\delta}
$$

are easily seen to provide better lower estimates for $\lambda$ (because $k \geq 4$ ). Thus the approximations $\lambda \simeq \kappa \simeq \varepsilon \simeq \alpha$ are really only of interest for non-simply connected regions.

Nonetheless, it is worthwhile to explicitly calculate the metric-densities $\kappa$ and $\tilde{\kappa}$ not only to obtain estimates for the best possible bilipschitz constants, but also to produce good estimates for the hyperbolic metric. As a simple example, if $z \in \Delta \subset \Omega$ and we can produce $a, b, c \in \partial \Delta \cap \partial \Omega$ with $\kappa_{\Delta}(z)=\lambda_{a b c}(z)$, then we easily obtain $\kappa_{\Omega}(z)=\lambda_{a b c}(z)$. Below we provide versions of this for the case when $\Delta$ is a disk.

4.A. Disks on $\hat{\mathbb{C}}$. In every disk the Ferrand and hyperbolic metrics agree, so

$$
\varepsilon(z)|d z|=\alpha(z)|d z|=\frac{1}{k} \varphi(z)|d z|=\frac{1}{k} \lambda(z)|d z| .
$$

What about $\kappa(z)|d z|$ ? There is an obvious conjecture as to what the extremal points must be for the center of the unit disk. We confirm this below; see Corollary 4.4.

Lemma 4.1. Suppose we have a collection of Möbius invariant conformal metrics $\rho_{\Delta}(z)|d z|$ defined for disks $\Delta \subset \hat{\mathbb{C}}$. Then there is a universal constant $C$ such that for all disks $\Delta \subset \hat{\mathbb{C}}, \rho_{\Delta}(z)|d z|=C \lambda_{\Delta}(z)|d z|$.

Proof. Fix a disk $\Delta$ in $\hat{\mathbb{C}}$ and a point $a \in \Delta$. Let $T: \Delta \rightarrow \mathbb{D}$ be a Möbius transformation with $T(a)=0$. Then Möbius invariance means that

$$
\rho_{\Delta}(z)|d z|=T^{*}\left[\rho_{\mathbb{D}}(\zeta)|d \zeta|\right]=\rho_{\mathbb{D}}(T z)\left|T^{\prime}(z)\right||d z| .
$$


Evaluating at $z=a$ produces

$$
\frac{\rho_{\Delta}(a)}{\lambda_{\Delta}(a)}=\left.\frac{\rho_{\Delta}(z)|d z|}{\lambda_{\Delta}(z)|d z|}\right|_{z=a}=\left.\frac{\rho_{\mathbb{D}}(T z)\left|T^{\prime}(z)\right||d z|}{\lambda_{\mathbb{D}}(T z)\left|T^{\prime}(z)\right||d z|}\right|_{z=a}=\frac{\rho_{\mathbb{D}}(0)}{\lambda_{\mathbb{D}}(0)} .
$$

Since $a \in \Delta$ was arbitrary, $\rho_{\Delta}(z)|d z|=C \lambda_{\Delta}(z)|d z|$, where $C=\rho_{\mathbb{D}}(0) / \lambda_{\mathbb{D}}(0)$.

An immediate corollary of the above is that for any disk in $\hat{\mathbb{C}}$,

$$
\kappa(z)|d z|=\frac{\kappa_{\mathbb{D}}(0)}{\lambda_{\mathbb{D}}(0)} \lambda(z)|d z| .
$$

We remark that the choices of the origin and the unit disk here and in the above lemma can be replaced, e.g., by any readily available point in any disk. In order to calculate $\kappa_{\mathbb{D}}(0)$, which then gives $\kappa(z)|d z|$ in any disk, it is fruitful to look first at the upper half plane.

Theorem 4.2. On the upper half-plane $\mathbb{H}$, the function $z \mapsto \lambda_{\mathbb{H}}(z) / \lambda_{01}(z)$ attains its minimum value uniquely at the point $\tau=e^{\pi i / 3}=(1+i \sqrt{3}) / 2$ and

$$
\frac{\lambda_{\mathbb{H}}(\tau)}{\lambda_{01}(\tau)}=h:=\frac{\sqrt{3} \Gamma^{6}(1 / 3)}{2^{2 / 3} 4 \pi^{3}} .
$$

Proof. It is convenient to consider the reciprocal function $u(z)=\lambda_{01}(z) / \lambda_{\mathbb{H}}(z)=$ $y \lambda_{01}(z)$, where $z=x+i y$. We first show that $u$ has limit 0 at each point of $\partial \mathbb{H}$ in $\hat{\mathbb{C}}$; this ensures that $u$ attains a maximum value inside $\mathbb{H}$.

It is elementary that $u(z) \rightarrow 0$ at each point $z$ of $\mathbb{R} \backslash\{0,1\}$, since $\lambda_{\mathbb{H}}(z)=1 / y \rightarrow$ $\infty$ at each point of this set. Next, $u(z)$ has limit 0 as $z \rightarrow 0$; this holds since $\mathbb{D}^{*} \subset \mathbb{C}_{01}$ gives

$$
u(z) \leq \frac{\lambda_{\mathbb{D}^{*}}(z)}{\lambda_{\mathbb{H}}(z)}=\frac{y}{|z| \log (1 /|z|)} \rightarrow 0 \text { as } z \rightarrow 0 .
$$

The function $u$ is invariant under $z \mapsto 1-\bar{z}$ (reflection across $\Re(z)=1 / 2$ ), so we also have $u(z) \rightarrow 0$ as $z \rightarrow 1$. In addition, $u$ is invariant under $z \mapsto 1 / \bar{z}$ (reflection over the unit circle), so $u(z) \rightarrow 0$ also as $z \rightarrow \infty$.

Because the positive function $u(z)=y \lambda_{01}(z)$ has vanishing boundary values, it must attain its maximum value at an interior point of the upper half-plane. Each point in $\mathbb{H}$ where $u$ attains its maximum value is a critical point. We show that $u$ has no critical points off the unit circle. Appealing to Fact 2.2 (d) we find that for $z=r e^{i \theta}$ with $0<r<1$,

$$
0<\lambda_{01}(z)+x \frac{\partial \lambda_{01}}{\partial x}(z)+y \frac{\partial \lambda_{01}}{\partial y}(z)=\frac{x}{y} \frac{\partial}{\partial x}\left[y \lambda_{01}\right](z)+\frac{\partial}{\partial y}\left[y \lambda_{01}\right](z) .
$$

This verifies that $u$ has no critical points in $\mathbb{D} \cap \mathbb{H}$. Since $r \lambda_{01}\left(r e^{i \theta}\right)$ is strictly decreasing on $(1, \infty)$ (see Fact 2.2 (d)), $u$ also has no critical points in $\mathbb{H} \backslash \overline{\mathbb{D}}$. Thus all critical points of $u$ must lie on the unit circle.

It now follows that the maximum value of $u$ must occur on $\{z:|z|=1\} \cap \mathbb{H}$. Because $u$ is invariant under $z \mapsto 1-\bar{z}$, we also find that the maximum value of $u$ can occur only on the circle $\{z:|z-1|=1\} \cap \mathbb{H}$. The unique point belonging to both of these semicircles is $\tau$. Therefore, $u$ attains its maximum value uniquely at $\tau=e^{\pi i / 3}$.

Theorem 4.3. For any disk or half-plane, $\kappa(z)|d z|=\frac{1}{h} \lambda(z)|d z|$. 
Proof. Thanks to Lemma 4.1, it suffices to establish this for the the upper half-plane $\mathbb{H}$. Since the quotient $\kappa_{\mathbb{H}} / \lambda_{\mathbb{H}}$ is invariant under all Möbius automorphisms of $\mathbb{H}$, we need only evaluate this quotient at the point $\tau$. Suppose $-\infty<a<b<c \leq \infty$. Let $T$ be the unique Möbius automorphism of $\mathbb{H}$ that satisfies $T(a)=0, T(b)=1$ and $T(c)=\infty$. The preceding theorem gives

$$
\frac{\lambda_{a b c}(\tau)}{\lambda_{\mathbb{H}}(\tau)}=\frac{\lambda_{01}(T(\tau))}{\lambda_{\mathbb{H}}(T(\tau))} \leq \frac{\lambda_{01}(\tau)}{\lambda_{\mathbb{H}}(\tau)}=\frac{1}{h}
$$

with equality if and only if $T$ fixes $\tau$.

Earlier we mentioned an obvious conjecture for the $\kappa$ extremal points for a disk. To see that we have corroborated this, we mention the unit disk analog of the above.

Corollary 4.4. For the unit disk $\mathbb{D}$,

$$
\kappa(0)=\sup _{a, b, c \in \mathbb{D}^{c}} \lambda_{a b c}(0)=\lambda_{1 \omega \omega^{2}}(0)=\frac{2}{h},
$$

where $\omega=e^{2 \pi i / 3}$. Moreover, $\kappa(0)=\lambda_{a b c}(0)$ if and only if $\{a, b, c\}$ is a rotation of $\left\{1, \omega, \omega^{2}\right\}$.

We remark that Betsakos Bet08, Theorem 1(b)] has recently proven this result via a different approach. We also note that Solynin established the stronger result [Sol99b, Theorem 4] that $\sup _{\zeta_{1}, \ldots, \zeta_{n} \in \mathbb{D}^{c}} \lambda_{\zeta_{1}, \ldots, \zeta_{n}}(0)$ is attained precisely when $\left\{\zeta_{1}, \ldots, \zeta_{n}\right\}$ is a rotation of $\left\{1, \omega, \ldots, \omega^{n-1}\right\}$ where $\omega=e^{2 \pi i / n}$. Solynin's proof makes extensive use of his polarization theory.

It is worthwhile to see how the above permits us to write down $\kappa$ for the upper half-plane. If we take the point at infinity as one of the extremal punctures, then we find that

$$
\kappa(z)=\lambda_{a b}(z)=\frac{1}{h y} \quad \text { where } a, b \in \mathbb{R} \text { are such that }|z-a|=|a-b|=|z-b| .
$$

Of course we can rotate these extremal points and so we also have, e.g.,

$$
\kappa(z)=\lambda_{a b c}(z)=\frac{1}{h y} \quad \text { where } z=x+i y, a=x-\sqrt{3} y, b=x, c=x+\sqrt{3} y .
$$

4.B. Slit plane. The correspondence $z=\zeta^{2}$ provides a conformal map from the right half-plane onto the slit plane $\mathbb{C} \backslash(-\infty, 0]$ from which we easily compute

$$
\forall r>0,|\vartheta|<\pi: \quad \lambda\left(r e^{i \vartheta}\right)=\frac{1}{2 r \cos (\vartheta / 2)} .
$$

We can use HIM08, Proposition 4.4] to compute the Ferrand metric. Here we determine the Gardiner-Lakic metrics.

Example 4.5. For the slit plane $\mathbb{C} \backslash[0, \infty)$,

$$
\kappa(z)=\tilde{\kappa}(z)= \begin{cases}\lambda_{0|z|}(z) & \text { when }|\operatorname{Arg}(z)| \geq \pi / 3 \\ \lambda_{a b}(z) & \text { when }|\operatorname{Arg}(z)| \leq \pi / 3\end{cases}
$$

where in the latter case $a, b \in[0, \infty)$ with $|z-a|=|a-b|=|z-b|$. 


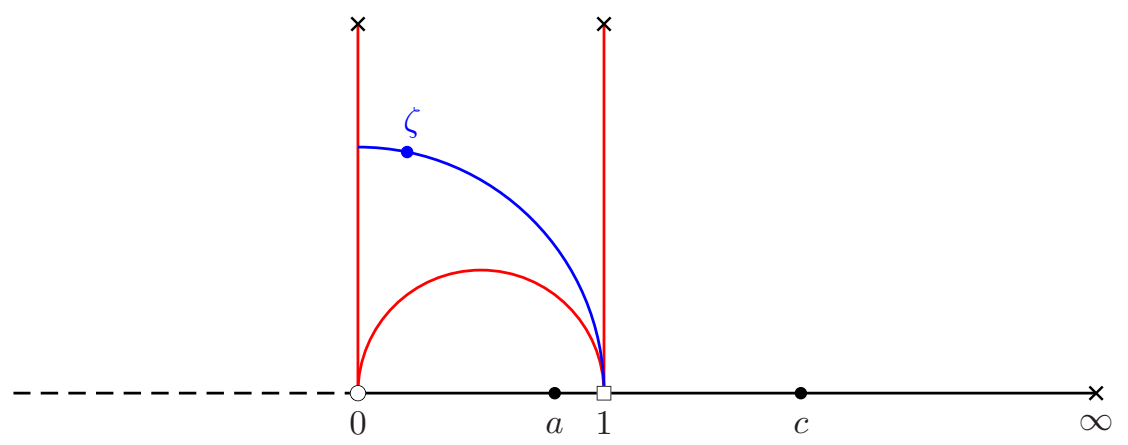

Figure 3. The $01 \infty$-picture

Proof. It suffices to prove the formula for $\kappa$. By dilation invariance and symmetry, it is enough to examine points $\zeta=e^{i \theta}$ with $\theta \in(0, \pi]$. When $\theta \leq \pi / 3$, we can find $a, b \in[0, \infty)$ with $|\zeta-a|=|a-b|=|\zeta-b|$. Then $\lambda_{a b}(\zeta)$ is the extremal value for the upper half-plane, and since this is a subdomain of $\mathbb{C} \backslash[0, \infty)$ it also gives $\kappa(\zeta)$. Thus we are left to deal with the case $\pi / 3<\theta \leq \pi$ (see Figure 3).

Let $0 \leq a<b<c \leq \infty$. We must demonstrate that $\lambda_{a b c}(\zeta) \leq \lambda_{01}(\zeta)$. In fact, we need only produce some $d \in(0, \infty)$ so that $\lambda_{a b c}(\zeta) \leq \lambda_{0 d}(\zeta)$. This is because

$$
\lambda_{0 d}(\zeta)=(1 / d) \lambda_{01}(\zeta / d) \leq \lambda_{01}(\zeta)
$$

the inequality above follows from Fact 2.2 (d).

When $\zeta$ lies in the closed left half-plane, we can reflect $a$ across the line $x=a / 2$ and then reflect $c$ across the circle $|z-c|=c$, and then appeal to Lemma 2.3 to deduce that

$$
\lambda_{a b c}(\zeta) \leq \lambda_{0 b c}(\zeta) \leq \lambda_{0 b}(\zeta)
$$

as desired. Thus it remains to consider the case when $\pi / 3<\theta<\pi / 2$.

First we confirm that we can either 'move' $c$ to $\infty$ or 'move' $a$ to 0 and obtain a value at least $\lambda_{a b c}(\zeta)$. Suppose $c \leq 1$. Then each of $a, b$, and $c$ lies in, while $\zeta$ lies outside, the closed disk $|z-c| \leq c$, so we can reflect $c$ across the circle $|z-c|=c$ and use Lemma 2.3 to obtain

$$
\lambda_{a b c}\left(e^{i \theta}\right) \leq \lambda_{a b}\left(e^{i \theta}\right) .
$$

Similarly, if $a \geq 1$, then $a / 2 \geq 1 / 2 \geq \Re(\zeta)$ so we can reflect $a$ across the line $x=a / 2$ and use Lemma 2.3 in conjunction with invariance under $z \mapsto 1 / \bar{z}$ (cf. Fact 2.2(a)) to conclude that

$$
\lambda_{a b c}(\zeta) \leq \lambda_{0 b c}(\zeta)=\lambda_{c^{-1} b^{-1}}(\zeta) .
$$

Thus if either $a \geq 1$ or $c \leq 1$, then we can find $0 \leq d<e<\infty$ with

$$
\lambda_{a b c}(\zeta) \leq \lambda_{d e}(\zeta) .
$$

Next, we assume that $a<1<c$ and verify that the above remains valid. It is convenient to look at the " $\omega^{2} 1 \omega$-picture" rather than the " $01 \infty$-picture"; here $\omega=e^{2 \pi i / 3}$ (see Figure 4). By this we mean to consider $\hat{\mathbb{C}} \backslash\left\{e^{i t}:|t| \leq 2 \pi / 3\right\}$ instead of $\mathbb{C} \backslash[0, \infty)$. Here the points $a<b<c$ correspond to $a^{\prime}=e^{i \alpha}, b^{\prime}=e^{i \beta}, c^{\prime}=e^{i \gamma}$ with $-2 \pi / 3 \leq \alpha<\beta<\gamma \leq 2 \pi / 3$, and $\zeta$ corresponds to $\zeta^{\prime} \in(\sqrt{3}-2,0)$. The hypotheses $a<1<c$ gives $\alpha<0<\gamma$. In fact, if necessary we can reflect $c^{\prime}$ across 


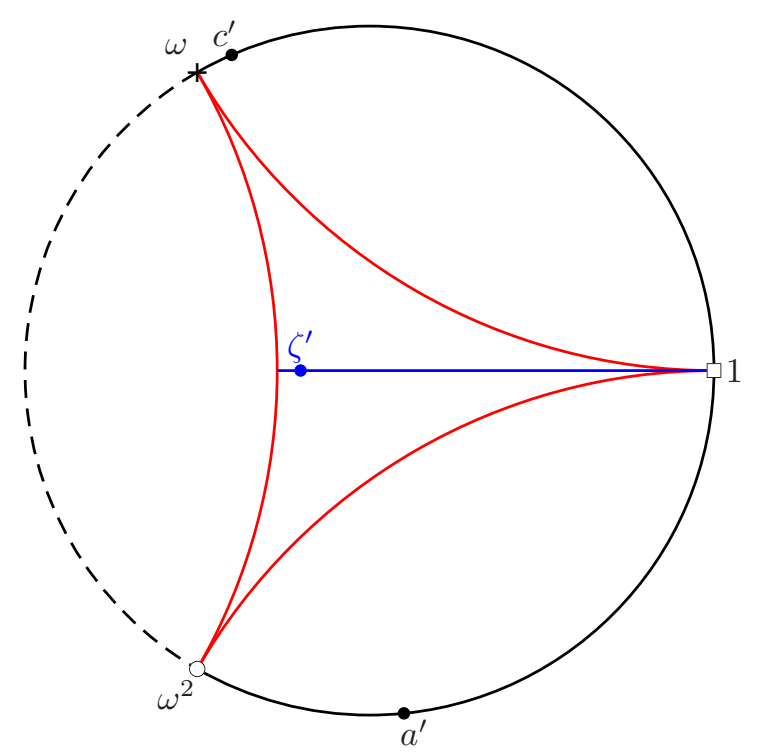

Figure 4 . The $\omega^{2} 1 \omega$-picture

the line $y=x$ and/or reflect $a^{\prime}$ across $y=-x$ and appeal to Lemma 2.3 to further assume that $\alpha \leq-\pi / 3$ and $\gamma \geq \pi / 3$.

Suppose $\gamma \geq|\alpha|$. Let $K$ be the unique circle through $\zeta^{\prime}$ with the property that $c^{\prime}$ and $\omega$ are symmetric with respect to $K$. (By mapping $\zeta^{\prime}$ to the origin via a disk automorphism, we easily see that such a circle $K$ exists.) Then $a^{\prime}, b^{\prime}, c^{\prime}$ all lie outside $K$, so another application of Lemma 2.3 provides us

$$
\lambda_{a b c}(\zeta)=\lambda_{a^{\prime} b^{\prime} c^{\prime}}\left(\zeta^{\prime}\right) \leq \lambda_{a^{\prime} b^{\prime} \omega}\left(\zeta^{\prime}\right)=\lambda_{a b}(\zeta)
$$

On the other hand, if $|\alpha| \geq \gamma$, a similar argument yields

$$
\lambda_{a b c}(\zeta)=\lambda_{a^{\prime} b^{\prime} c^{\prime}}\left(\zeta^{\prime}\right) \leq \lambda_{\omega^{2} b^{\prime} c^{\prime}}\left(\zeta^{\prime}\right)=\lambda_{0 b c}(\zeta)=\lambda_{c^{-1} b^{-1}}(\zeta) .
$$

Thus in all cases, given $0 \leq a<b<c \leq \infty$, we have produced $0 \leq d<e<\infty$ with

$$
\lambda_{a b c}(\zeta) \leq \lambda_{d e}(\zeta) \quad \text { where } \zeta=e^{i \theta} \text { with } \pi / 3<\theta<\pi / 2 .
$$

Therefore, it remains to demonstrate that for given $0 \leq a<b<\infty$, there exists a $d \in(0, \infty)$ such that

$$
\lambda_{a b}(\zeta) \leq \lambda_{0 d}(\zeta)
$$

So, let $0 \leq a<b<\infty$ be given. One more call to Lemma 2.3. and use of symmetry, permits us to assume that $a \leq \Re(\zeta) \leq(a+b) / 2$. Suppose first that $d:=b-a \geq|\zeta-a|$. Put $w=\zeta-a$. According to Fact 2.2(e), $\lambda_{0 d}(x+i y)$ is an increasing function of $x$ for $x+i y \in[w, \zeta]$, so

$$
\lambda_{a b}(\zeta)=\lambda_{0 d}(w) \leq \lambda_{0 d}(\zeta)
$$


as desired. Finally, suppose $b-a<|\zeta-a|$ and let $d:=a+|\zeta-a|$ and $e:=d-a=$ $|\zeta-a|$. We claim that

$$
\lambda_{a b}(\zeta) \leq \lambda_{a d}(\zeta) \leq \lambda_{0 e}(\zeta)
$$

The second inequality above follows from the first case considered. To prove the first inequality, we may assume that $a=0, b=1, d=|\zeta|>1$. Since $|z| \lambda_{01}(|z|)$ decreases as $|z|>1$ increases (cf. Fact $2.2(\mathrm{~d})$ ), $|\zeta| \lambda_{01}(\zeta) \leq \lambda_{01}(\zeta /|\zeta|)$. Thus, as asserted,

$$
\lambda_{01}(\zeta) \leq \frac{1}{|\zeta|} \lambda_{01}\left(\frac{\zeta}{|\zeta|}\right)=\frac{1}{d} \lambda_{01}\left(\frac{\zeta}{d}\right)=\lambda_{0 d}(\zeta) .
$$

4.C. Infinite strip. For the infinite strip $\{x+i y:|y|<\pi / 2\}$,

$$
\lambda(x+i y)=\sec (y) \quad \text { and } \quad \varphi(x+i y)=\frac{\pi}{(\pi / 2)^{2}-y^{2}} .
$$

A conformal map from the right half-plane is given by $z=\log (\zeta)$ (the principal branch of the logarithm). Since the strip is simply connected,

$$
\varepsilon=\alpha=\frac{\varphi}{k} \quad \text { and } \quad \tilde{\varepsilon}=\tilde{\alpha}=\frac{\tilde{\varphi}}{k}=\frac{1}{k \delta} .
$$

It is straightforward to calculate $\tilde{\kappa}$. For notational convenience, we first make an affine change of variables.

Example 4.6. For the infinite strip $\Sigma:=\{x+i y: 0<y<1\}$,

$$
\tilde{\kappa}(z)= \begin{cases}\lambda_{01}(\delta(z)) & \text { when } \delta(z) \geq t, \\ {[h \delta(z)]^{-1}} & \text { when } 0<\delta(z) \leq t\end{cases}
$$

where $t$ is the unique number in $(0,1 / 2)$ satisfying $t \lambda_{01}(t)=1 / h$.

Proof. By translation invariance and symmetry, it suffices to consider points iy with $y \in(0,1 / 2]$. Thus we seek to determine $\tilde{\kappa}(i y)=\max _{a, b \in \partial \Sigma} \lambda_{a b}(i y)$. Our first goal is to confirm that

$$
\tilde{\kappa}(i y)=\max \left\{\lambda_{01}(y), 1 /(h y)\right\} .
$$

If both $a, b$ were on the line $\Im(z)=1$, then we could reflect both points across $\Im(z)=1 / 2$ and appeal to Lemma 2.3 to obtain a larger value; so $\Im(a)=1=\Im(b)$ does not yield an extremal configuration. When both $\Im(a)=0=\Im(b)$, the extremal configuration is the same as for the upper half-plane; that is,

$$
\max _{a, b \in \mathbb{R}} \lambda_{a b}(i y)=\frac{\sqrt{3}}{2 y} \lambda_{01}(\tau)=\frac{\sqrt{3}}{2 y} \cdot \frac{2}{\sqrt{3} h}=\frac{1}{h y}=\frac{1}{h \delta(i y)} .
$$

Suppose $a, b$ lie on opposite edges of $\Sigma$. We show below that here the extremal configuration arises precisely when $\{a, b\}=\{0, i\}$; that is,

$$
\max _{\Im(a)=0, \Im(b)=1} \lambda_{a b}(i y)=\lambda_{0 i}(i y)=\lambda_{01}(y) .
$$

Assuming the above, we have now verified that $\tilde{\kappa}(i y)=\max \left\{\lambda_{01}(y), 1 /(h y)\right\}$.

We note that $\lambda_{01}(1 / 2)=4 / k>2 / h$ which tells us that the extremal punctures for the point $i / 2$ are indeed $0, i$. Now we examine what happens as $y \searrow 0$. Recall that $x \lambda_{01}(x)$ is strictly increasing for $x \in(0,1)$; see Fact $2.2(\mathrm{~d})$. As $\mathbb{D}^{*} \subset \mathbb{C}_{01}$, $x \lambda_{01}(x) \leq x \lambda_{\mathbb{D}^{*}}(x)=1 /|\log x| \rightarrow 0$ as $x \rightarrow 0$. It follows that there is a unique $t \in(0,1 / 2)$ satisfying $t \lambda_{01}(t)=1 / h$. Then for $0<x<t$ (or $\left.t<x \leq 1 / 2\right)$ we 
have $\lambda_{01}(x)<1 / h x$ (or $\lambda_{01}(x)>1 / h x$, respectively); this now yields the asserted formula for $\tilde{\kappa}(i y)$.

It remains to establish (4.7). We examine several cases which depend on the locations of $a, b \in \partial \Sigma$. In each case we use Fact 2.4 and/or Lemma2.3 to corroborate that $\lambda_{a b}(i y) \leq \lambda_{0 i}(i y)$. First, suppose $a=0$; the case $b=i$ is handled in a similar manner. Let $v=y+|b-i y|$. According to Facts 2.4(c) (and then (a)),

$$
\lambda_{0 b}(i y) \leq \lambda_{0, i v}(i y) \leq \lambda_{0 i}(i y) \text {. }
$$

Next, suppose $a \Re(b)>0$; say both are positive. Pick the point, $a$ or $b$, which has the smallest real part, say $r$, and reflect this point across the line $\Re(z)=r / 2$. Lemma 2.3 says that this will give us a larger value, and now we are back in the first case.

Third, suppose $a \neq 0$ and $b \neq i$ are such that $i y \in[a, b]$. Rotating about $i y$ we see that

$$
\lambda_{a b}(i y)=\lambda_{i u, i v}(i y) \quad \text { where } \quad u=y-|a-i y| \text { and } v=y+|b-i y| .
$$

Then two applications of Fact 2.4(a) produce

$$
\lambda_{i u, i v}(i y) \leq \lambda_{i u, i}(i y) \leq \lambda_{0 i}(i y) .
$$

Finally, suppose $a \Re(b)<0$; say, $a<0<\Re(b)$. Let $L$ be the line through $a$ and $i y$. Assume that $b$ lies 'below and to the right' of $L$. We rotate $b$ about iy 'up' to the point $c \in L$ and then project $c$ 'down' and along $L$ to get the point $d \in L \cap\{\Im(z)=1\}$. By Facts 2.4 (c) (and then (a)) we now have

$$
\lambda_{a b}(i y) \leq \lambda_{a c}(i y) \leq \lambda_{a d}(i y)
$$

and since $a, i y, d$ are collinear, we are back to the third case just above.

If $b$ does not lie 'below and to the right' of $L$, then $a$ must lie 'above and to the left' of the line through $b$ and $i y$, and then a similar argument applies.

4.D. Punctured disk. Another simple, although important, example is the punctured unit disk $\mathbb{D}^{*}:=\mathbb{D} \backslash\{0\}$ for which

$$
\lambda(z)=[|z||\log | z||]^{-1} \quad \text { and } \quad \varphi(z)=[|z|(1-|z|)]^{-1} ;
$$

a holomorphic covering from the right half-plane to $\mathbb{D}^{*}$ is provided by $z=e^{-\zeta}$. We can also calculate four of the metrics in $\mathbb{D}^{*}$. Note in particular that this provides an example where $\tilde{\alpha} \neq \tilde{\varepsilon}$.

Example 4.8. For the punctured unit disk $\mathbb{D}^{*}$ we have:

$$
\alpha(z)=\varepsilon(z)= \begin{cases}\varphi(z) / k & \text { when } 1 / 3 \leq|z|<1 \\ \varphi(z) /\left[k+\log \frac{1-|z|}{2|z|}\right] & \text { when } 0<|z| \leq 1 / 3 .\end{cases}
$$

On the other hand,

$$
\tilde{\alpha}(z)= \begin{cases}{[k(1-|z|)]^{-1}} & \text { when } 1 / 2 \leq|z|<1, \\ {\left[|z| \log \left(e^{k} /|z|\right)\right]^{-1}} & \text { when } 0<|z|<1 / 2,\end{cases}
$$

whereas

$$
\tilde{\varepsilon}(z)= \begin{cases}{[k(1-|z|)]^{-1}} & \text { when } t \leq|z|<1, \\ {\left[|z| \log \left(e^{k} /|z|\right)\right]^{-1}} & \text { when } 0<|z| \leq t,\end{cases}
$$

where $t \in(1 / e, 1 / 2)$ is the unique solution to $x \log (1 / x)=k(1-2 x)$. 
Proof. Thanks to rotational symmetry it suffices to consider points $z=|z|=x \in$ $(0,1)$. It is transparent that, for $1 / 2 \leq x \leq 1$, the Apollonian circle through $x$ with limit points 0 and 1 meets $\partial \mathbb{D}$; an easy calculation confirms that this continues to hold for $1 / 3 \leq x \leq 1$. Thus for these points, $\gamma(x)=0$ and $\alpha(x)=\varphi(x) / k$. For $0<x<1 / 3$, the largest Möbius annulus in $\mathbb{D}^{*}$ symmetric about $C(x ; 0,1)$ will be tangent to $\partial \mathbb{D}$ at -1 and so we find that $\gamma(x)=\log [(1-x) / 2 x]$. This gives the formula for $\alpha(x)$.

Now we determine $\varepsilon(x)$ for $x \in(0,1 / 3)$. For boundary points $a, b \in \partial \mathbb{D}$ we have $\gamma(\cdot ; a, b) \equiv 0$ and so the largest of the values $\tau_{a b}(x) /[k+\gamma(x ; a, b)]$ for such $a, b$ is $\varphi(x) / k=2 /\left[k\left(1-x^{2}\right)\right]$. On the other hand, for $a=0$ and $b \in \partial \mathbb{D}$,

$$
\gamma(x ; 0, b)=\gamma(r b ; 0, b)=\gamma(r ; 0,1)=\log \frac{1-r}{2 r}=\log \frac{|x-b|}{2 x},
$$

where $r>0$ is such that $r b \in C(x ; 0, b)$ (so, $r=x /(x+|x-b|))$. It is easy to check that

$$
\left(x(1-x)\left[k+\log \frac{1-x}{2 x}\right]\right)^{-1}=\frac{\tau_{01}(x)}{k+\gamma(x ; 0,1)}=\sup _{b \in \partial \mathbb{D}} \frac{\tau_{0 b}(x)}{k+\gamma(x ; 0, b)}>\frac{2}{k\left(1-x^{2}\right)}
$$

and thus $\varepsilon(x)=\alpha(x)$.

It is straightforward to check that

$$
\beta(z)=\tilde{\gamma}(z)= \begin{cases}0 & \text { when } 1 / 2 \leq|z|<1, \\ |\log | z|| & \text { when } 0<|z|<1 / 2,\end{cases}
$$

which establishes the asserted formula for $\tilde{\alpha}(z)$. To determine $\tilde{\varepsilon}(z)$ we note that $\tilde{\gamma}(z ; 0)=|\log | z||$ whereas for each $a \in \partial \mathbb{D}, \tilde{\gamma}(z ; a)=0$. Using rotational symmetry we may assume that $z=|z|=x \in(0,1)$, and then

$$
\tilde{\varepsilon}(x)=1 / \min \{k(1-x), x(k+\log 1 / x)\}
$$

which yields the given formula.

While we cannot determine either $\kappa$ or $\tilde{\kappa}$ for $\mathbb{D}^{*}$, we do have the following information. The origin is always an extremal puncture for $\kappa$, i.e., $\kappa_{\mathbb{D}^{*}}>\kappa_{\mathbb{D}}$, but this is not the case for $\tilde{\kappa}$. Also, we do know $\tilde{\kappa}(z)$ for $z$ near 0 as well as for $z$ near $\partial \mathbb{D}$.

Remarks 4.9. (a) For all $z \in \mathbb{D}^{*}, \kappa(z)=\sup _{|a|=1=|b|} \lambda_{a b 0}(z)$.

(b) There are $0<s \leq t<1$ such that for $z \in \mathbb{D}^{*}$,

$$
\tilde{\kappa}(z)= \begin{cases}\lambda_{01}(|z|) & \text { when } 0<|z| \leq s \\ \tilde{\kappa}_{\mathbb{D}}(z) & \text { when } t \leq|z|<1 .\end{cases}
$$

Proof. To establish (a) it suffices to confirm that $\kappa_{\mathbb{D}}^{*}>\kappa_{\mathbb{D}}$. By Möbius invariance, we may consider the upper half-plane $\mathbb{H}$ punctured at $i$ and $z=i y$ with $y \in(0,1)$. Start with any distinct $a, b, c \in \partial \mathbb{H}$; maximizing $\lambda_{a b c}(z)$ produces $\kappa_{\mathbb{H}}(z)$ which we know how to calculate. Taking the point at infinity as one of the extremal punctures we find that $\kappa_{\mathbb{H}}(z)=\lambda_{-a a}(z)$ where $a>0$ is such that $|z-a|=2 a$. Now we reflect the point at infinity across the circle $\partial D(i ;|a-i|)$ and appeal to Lemma 2.3 to produce a larger value, $\lambda_{-a a i}(z)>\lambda_{-a a}(z)$. It follows that $\kappa_{\mathbb{H} \backslash\{i\}}(z)>\kappa_{\mathbb{H}}(z)$.

Next we corroborate (b). Clearly for $z \in \mathbb{D}^{*}$ sufficiently close to the origin, the origin must be an extremal puncture for $\tilde{\kappa}$ (because for $a, b \in \partial \mathbb{D}, \lambda_{a b}(0)$ is finite). E.g., for all $a, b \in \partial \mathbb{D}$ we have $\lambda_{a b}(0) \leq 2$, so there is an $s>0$ such that for all 
$|z| \leq s, \lambda_{a b}(z) \leq 3$. It thus follows that $\tilde{\kappa}(z)=\lambda_{01}(|z|)$ for $0<|z| \leq s$. On the other hand, when $|z| \rightarrow 1$, we know that $\tilde{\kappa}(z) \simeq \lambda(z)$ which blows up like $(1-|z|)^{-1}$. However, $\lambda_{01}(|z|)$ only blows up like $[(1-|z|)|\log (1-|z|)|]^{-1}$ and so it is not 'big enough'.

4.E. Annuli. Finally, we take a look at the annulus $A(R):=\{z: 1 / R<|z|<R\}$. A holomorphic covering from the right half-plane to $A(R)$ is given by $z=\zeta^{i t}=$ $e^{i t \log \zeta}$ where $\log R=t \pi / 2$. A routine computation produces

$$
\lambda_{A(R)}(z)=\frac{\pi / 2}{|z| \log R} \sec \left(\frac{\pi}{2} \frac{\log |z|}{\log R}\right) \quad \text { and } \quad \varphi_{A(R)}(z)=\frac{R-1 / R}{(R-|z|)(|z|-1 / R)} .
$$

To calculate the Ferrand metric we can use [HIM08, Proposition 4.4], however it is more straightforward to appeal to [HIM08, §3.D and Lemma 4.7]. We note in particular that

$$
\lambda_{A(R)}(1)=\frac{\pi / 2}{\log R}=\frac{\pi}{\bmod A(R)}
$$

where $\bmod A(R)=2 \log R$ is the conformal modulus of $A(R)$.

We can determine $\alpha$ and $\tilde{\alpha}$ for an annulus once we know $\gamma$ and $\beta$. Both $\gamma$ and $\beta$ are zero at many points in an annulus, but $\gamma$ is never identically zero whereas $\beta \equiv 0$ in any thin annulus having conformal modulus at most $\log 5$.

Example 4.10. For the annulus $A:=\{z: 1<|z|<R\}$,

$$
\beta(z)= \begin{cases}0 & \text { when } R \leq 5, \text { or if } R>5 \text { and }|z| \in(1,3] \cup[(R+1) / 2, R), \\ \log \frac{\delta(z)}{2} & \text { when } 5<R \leq 9 \text { and } 3<|z|<(R+1) / 2, \\ \log \frac{R-1}{\delta(z)} & \text { or, if } R>9 \text { and } 3<|z| \leq 1+\sqrt{2(R-1)}\end{cases}
$$

and

$$
\gamma(z)= \begin{cases}0 & \text { when }|z| \in\left(1, t_{1}\right] \cup\left[t_{2}, R\right), \\ \log \frac{R+1}{2} \frac{|z|-1}{R-|z|} & \text { when } t_{1}<|z| \leq \sqrt{R}, \\ \log \frac{R+1}{2 R} \frac{R-|z|}{|z|-1} & \text { when } \sqrt{R} \leq|z| \leq t_{2},\end{cases}
$$

where $t_{1}=(3 R+1) /(R+3)$ and $t_{2}=R(R+3) /(3 R+1)$. (Notice that we always have $1<t_{1}<\sqrt{R}<t_{2}<(R+1) / 2$.)

Proof. Thanks to rotational symmetry it suffices to consider points $z=|z|=x \in$ $(1, R)$. The computation for $\beta(x)$ is straightforward and left to the reader. It is transparent that for $(R-1) / 2 \leq x<R$, the Apollonian circle through $x$ with limit points 1 and $R$ meets $\partial A$; an easy calculation confirms that this continues to hold for $t_{2} \leq x \leq R$. Thus for these points, $\gamma(x)=0$ (and so $\left.\alpha(x)=\varphi(x) / k\right)$. Similarly we have $\gamma(x)=0$ for $1<x \leq t_{1}$ (see Figure 5). For $t_{1}<x<t_{2}$, the largest Möbius annulus in $A$ symmetric about $C(x ; 1, R)$ will be tangent to $\partial A$ either at -1 or at $-R$, and this gives the asserted formula for $\gamma(x)$. 


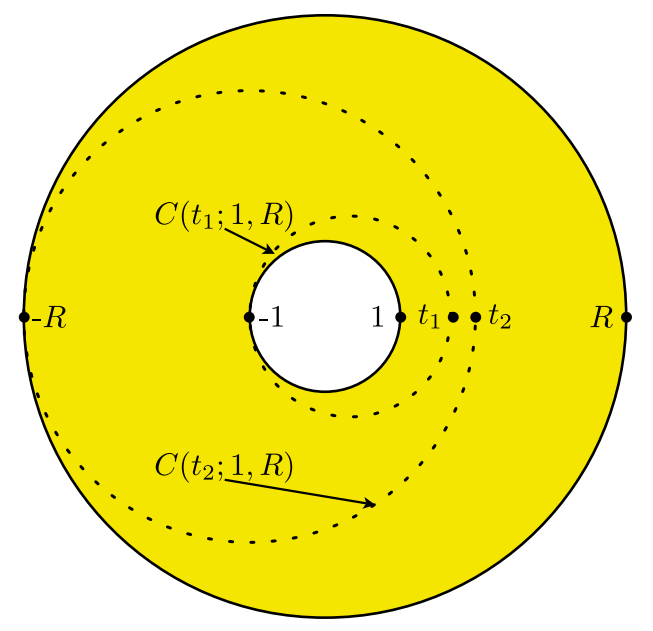

Figure 5. Apollonian circles in the annulus $\{1<|z|<R\}$

\section{Proofs of MAin Results}

Here we present proofs of Theorem $\mathrm{A}$ and Corollaries B, $\mathrm{C}$ and $\mathrm{D}$ see 5.4 and 5.B. First we discuss some general inequalities which hold among the metrics studied herein. Then we describe certain bilipschitz constants. We conclude by demonstrating that all six metrics studied here are quasiinvariant with respect to holomorphic covering maps.

5.A. The basic inequalities. There is a general framework of inequalities which all our metrics satisfy.

Theorem 5.1. For any hyperbolic region $\Omega \subset \hat{\mathbb{C}}$ we always have

$$
\alpha \leq \varepsilon \leq \kappa \leq \lambda \text { and } \varepsilon \leq \frac{\varphi}{k} .
$$

When $\Omega \subset \mathbb{C}$ is a hyperbolic plane region, we also obtain

$$
\tilde{\alpha} \leq \tilde{\varepsilon} \leq \tilde{\kappa} \leq \lambda, \quad \tilde{\varepsilon} \leq \frac{1}{k \delta}, \quad \tilde{\varepsilon} \leq \varepsilon, \quad \text { and } \quad \tilde{\kappa} \leq \kappa .
$$

Proof. There is a simple method to obtain a lower bound on the hyperbolic metric in any hyperbolic region $\Omega \subset \hat{\mathbb{C}}$. Fix a point $z \in \Omega$. By Möbius invariance, we may assume that $z \in \mathbb{C}$. If $\Omega$ contains the point at infinity, we must use a local coordinate there and remember that we are dealing with metrics.

Let $a, b, c$ be three distinct points in $\Omega^{c}$. Then $\Omega \subset \hat{\mathbb{C}}_{a b c}$, so domain monotonicity gives

$$
\lambda_{a b c}(z) \leq \lambda_{\Omega}(z) .
$$

Taking the supremum over all triples in the complement of $\Omega$ yields $\kappa(z) \leq \lambda(z)$.

As we mention in the Introduction, even if we can determine the extremal points $a, b, c$ giving $\kappa(z)$, we are then confronted with calculating (or estimating) $\lambda_{a b c}$. Fortunately, there is an explicit easily calculated lower bound for $\lambda_{a b c}(z)$ which is obtained by making the change of variables $w=[z, a, b, c]$ and then appealing to 
parts (b) and (c) of Fact 2.2. We find that

$$
\frac{|a-b|}{|z-a||z-b|} \cdot \frac{1}{k+|\log | z, a, b, c||} \leq \lambda_{a b c}(z) .
$$

Taking the supremum over all $a, b, c \in \Omega^{c}$ we deduce that $\varepsilon(z) \leq \kappa(z)$.

Now recall that $\varepsilon(z)$ can also be computed by maximizing $\tau_{a b}(z) /[k+\gamma(z ; a, b)]$ over all distinct $a, b \in \partial \Omega$. Likewise, in the definition of $\alpha(z)$ we also consider such a supremum, but here we only look at points $a, b \in \partial \Omega$ with $\tau_{a b}(z)=\varphi(z)$. Thus we readily obtain $\alpha(z) \leq \varepsilon(z)$. Moreover, since $\gamma(z ; a, b) \geq 0$ and $\tau_{a b}(z) \leq \varphi(z)$, it is transparent that $\varepsilon(z) \leq \varphi(z) / k$.

When $\Omega \subset \mathbb{C}$, similar reasoning applies to the related metrics $\tilde{\alpha}(z)|d z|, \tilde{\varepsilon}(z)|d z|$ and $\tilde{\kappa}(z) \mid d z$. In this setting it is elementary that $\tilde{\kappa} \leq \kappa, \tilde{\varepsilon} \leq \varepsilon$ and $\tilde{\varepsilon} \leq 1 /(k \delta)$.

A natural question to ask is when equality may hold in any of the above inequalities.

Proposition 5.2. Let $\Omega$ be a hyperbolic region, either in $\hat{\mathbb{C}}$ or in $\mathbb{C}$ as needed. There exists a point $z \in \Omega$ with:

(a) $\kappa(z)=\lambda(z)$ if and only if $\Omega$ is a thrice punctured sphere;

(b) $\tilde{\kappa}(z)=\lambda(z)$ if and only if $\Omega$ is a twice punctured plane;

(c) $\varepsilon(z)=\kappa(z)=\lambda_{a b c}(z)$ with $a, b, c \in \Omega^{c}$ if and only if $z$ lies on the circle through $a, b, c$ and either $[z, a, b, c]=1$ or $[z, b, c, a]=1$ or $[z, c, b, a]=1$;

(d) $\tilde{\varepsilon}(z)=\tilde{\kappa}(z)=\lambda_{a b}(z)$ with $a, b \in \Omega^{c}$ if and only if $z$ lies on the line through $a, b$ and either $z=2 a-b$ or $z=2 b-a$.

Proof. These statements concerning equality holding at some point $z$ are straightforward consequences of Facts 2.1 and 2.2

It is useful to understand when $\gamma(z)=0$ or $\beta(z)=0$. It is easy to see that in any simply connected region, $\gamma \equiv 0$ and $\beta \equiv 0$. However, neither $\gamma \equiv 0$ nor $\beta \equiv 0$ imply simple connectivity. Indeed, in any round annulus with modulus at most $\log 5$ we have $\beta \equiv 0$; see 4 .E. While $\gamma$ is never identically zero in any round annulus, it is so in any ring domain which cannot be separated by a circle; e.g., in the non-simply connected region $\Omega=\{x+i y: 0<x<4,|y|<1\} \backslash[1,3]$ we do have $\gamma \equiv 0$. We also have the following information.

Lemma 5.3. Let $\Omega$ be a hyperbolic region. For $z \in \Omega \subset \hat{\mathbb{C}}$, the following conditions are equivalent:
(a) $\gamma(z)=0$.
(b) $\alpha(z)=\varphi(z) / k$.
(c) $\varepsilon(z)=\varphi(z) / k$.

For $z \in \Omega \subset \mathbb{C}$, the following conditions are equivalent:

(a) $\beta(z)=0$.

(官) $\tilde{\alpha}(z)=1 /[k \delta(z)]$.

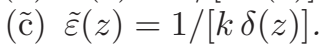

Proof. It is easy to see that (a) implies both (b) and (c) and that (b) implies (a); similarly $(\tilde{\mathrm{a}}) \Leftrightarrow(\tilde{\mathrm{b}}) \Rightarrow(\tilde{\mathrm{c}})$. If (c) holds, then by choosing the appropriate points $a, b \in \partial \Omega$ we obtain

$$
\frac{\varphi(z)}{k}=\varepsilon(z)=\frac{\tau_{a b}(z)}{k+\gamma(z ; a, b)} \leq \frac{\varphi(z)}{k+\gamma(z ; a, b)} \leq \frac{\varphi(z)}{k} ;
$$


the rightmost equality above forces $\gamma(z ; a, b)=0$ which then gives $\tau_{a b}(z)=\varphi(z)$ and so $\gamma(z)=0$. A similar argument reveals that $(\tilde{\mathrm{c}})$ implies $(\tilde{\mathrm{a}})$.

\subsection{Proof of Theorem $\mathrm{A}$.}

We confirm that for any hyperbolic region $\Omega$ in $\hat{\mathbb{C}}$, and for all $z \in \Omega$,

$$
\frac{\varphi(z)}{k+\gamma(z)} \leq \lambda(z) \leq \frac{\pi}{2} \frac{\varphi(z)}{\gamma(z)}
$$

According to Theorem 5.1, $\alpha \leq \lambda$, which gives the lower bound on $\lambda(z)$. To establish the upper bound for $\lambda(z)$, we may assume that $z \in \Omega \cap \mathbb{C}$ and that $\gamma(z)>0$. According to $\S \$ 3$.B and 3 .C . we can select points $a, b \in \partial \Omega$ with $\varphi(z)=\tau_{a b}(z)$ and $\gamma(z)=\gamma(z ; a, b)$. Next, put $\gamma=\gamma(z)$ and let

$$
A=A(z ; a, b, \gamma)=\left\{\zeta \in \hat{\mathbb{C}}: e^{-\gamma}<|z, a, b, \zeta|<e^{\gamma}\right\}
$$

be the Möbius annulus which is symmetric with respect to $C(z ; a, b)$ (the Apollonian circle through $z$ with limit points $a, b$ - see the end of $2 . \mathrm{A}$ ) and has conformal $\operatorname{modulus} \bmod (A)=2 \gamma(z)$. Note that $A \subset \Omega$.

To calculate $\lambda_{A}(z)$, we consider the Möbius change of variables

$$
w=\frac{z-b}{z-a} \frac{\zeta-a}{\zeta-b} .
$$

Then

$$
\left|\frac{d w}{d \zeta}\right|_{\zeta=z} \mid=\frac{|a-b|}{|z-a||z-b|}=\tau_{a b}(z)=\varphi(z) .
$$

Now $\zeta=z$ corresponds to $w=1$, and the image of $A$ is a round annulus, so the information in 4 .E reveals that

$$
\lambda_{A}(z)=\frac{\pi}{2} \frac{\varphi(z)}{\gamma(z)} .
$$

Finally, since $A \subset \Omega$, domain monotonicity of the hyperbolic metric tells us that

$$
\lambda(z) \leq \lambda_{A}(z)=\frac{\pi}{2} \frac{\varphi(z)}{\gamma(z)}
$$

as asserted.

5.B. Bilipschitz constants. Given a collection of metrics $\rho(z)|d z|$ defined for hyperbolic domains (in $\mathbb{C}$ or in $\hat{\mathbb{C}}$ ) and always satisfying $\rho \leq \lambda \leq C \rho$, we write $K_{\rho}$ for the best (i.e., smallest) such constant $C$. Similarly, we let $\bar{K}_{\rho}^{\mathrm{sc}}$ be defined as for $K_{\rho}$ but restricting the hyperbolic regions to be simply connected. Thus,

$$
K_{\rho}:=\sup _{\Omega} \sup _{z \in \Omega} \frac{\lambda(z)|d z|}{\rho(z)|d z|} \quad \text { and } \quad K_{\rho}^{\mathrm{sc}}:=\sup _{\Omega \text { s.c. }} \sup _{z \in \Omega} \frac{\lambda(z)|d z|}{\rho(z)|d z|}
$$

where $\Omega$ is a hyperbolic region in $\hat{\mathbb{C}}$ (or in $\mathbb{C}$ ); $K_{\rho}$ is the best bilipschitz constant for the identity map between hyperbolic geometry and $\rho$-geometry. For example, the classical inequalities

$$
\frac{1}{2 \delta} \leq \frac{\varphi}{2} \leq \lambda \leq \varphi \leq \frac{2}{\delta}
$$

in conjunction with knowledge of these metrics at $z=0$ in $\mathbb{D}($ e.g., $\lambda(0)=2=\varphi(0)$ ) tell us that

$$
K_{\varphi / 2}^{\mathrm{sc}}=2 \quad \text { and similarly } \quad K_{1 / 2 \delta}^{\mathrm{sc}}=4
$$


Clearly $K_{\rho} \geq K_{\rho}^{\mathrm{sc}}$, and when $\rho \leq \sigma \leq \lambda \leq C \rho$ we have $K_{\rho} \geq K_{\sigma}$. Thus an immediate consequence of the basic inequalities in Theorem 5.1 is that

$$
\begin{aligned}
K_{\alpha} & \geq K_{\varepsilon} \geq K_{\kappa}, \\
K_{\tilde{\alpha}} & \geq K_{\tilde{\varepsilon}} \geq K_{\tilde{\kappa}}, \\
K_{\tilde{\varepsilon}} & \geq K_{\varepsilon} \text { and } \quad K_{\tilde{\kappa}} \geq K_{\kappa} .
\end{aligned}
$$

In addition, our knowledge of these metrics at the origin in the unit disk $\mathbb{D}$ (see 4.A. Corollary 4.4 and [SV05, Remark 3.2]) tells us that

$$
K_{\varepsilon}^{\mathrm{sc}} \geq k, \quad K_{\tilde{\varepsilon}}^{\mathrm{sc}} \geq 2 k, \quad K_{\kappa}^{\mathrm{sc}} \geq h, \quad K_{\tilde{\kappa}}^{\mathrm{sc}} \geq k .
$$

Proof of Corollaries B, C, and D, We first verify Corollary B, Thanks to Theorem 5.1 it remains only to demonstrate that for any hyperbolic region $\Omega \subset \hat{\mathbb{C}}$,

$$
\lambda \leq(k+\pi / 2) \alpha .
$$

This is a result of combining the 'classical' inequality $\lambda \leq \varphi$ (see the end of 8 2.C) together with the inequality $\lambda \leq(\pi / 2)(\varphi / \gamma)$ from Theorem $\mathrm{A}$. Indeed, we obtain

$$
\lambda \leq \varphi \min \left\{1, \frac{\pi}{2} \frac{1}{\gamma}\right\}=\frac{\varphi}{k+\gamma} \min \left\{k+\gamma, \frac{\pi}{2}\left(1+\frac{k}{\gamma}\right)\right\} \leq(k+\pi / 2) \alpha .
$$

Notice that (2.5) gives a similar result involving $\tilde{\alpha}(z)|d z|$; here the best absolute constant $C$ satisfies $2 k \leq C \leq 2 k+\pi / 2$ and the 2 arises from the classical bound $\lambda \leq 2 / \delta$. (For simply connected regions we can take $C=2 k$.)

We have thus established that

$$
K_{\alpha} \leq k+\pi / 2 \quad \text { and } \quad K_{\tilde{\alpha}} \leq 2 k+\pi / 2 .
$$

From the comments preceding this proof, we now have

$$
h \leq K_{\kappa} \leq K_{\alpha} \leq k+\pi / 2 \text { and } k \leq K_{\varepsilon} \leq K_{\alpha} \leq k+\pi / 2 .
$$

All the inequalities in Corollaries B, C] and D now follow. Notice that the lower bounds for our 'best bilipschitz constants' arise from knowing the metrics at the origin in $\mathbb{D}$.

It remains to corroborate the asserted inequalities for simply connected hyperbolic domains. In a simply connected hyperbolic region in $\hat{\mathbb{C}}$,

$$
\alpha=\varepsilon=\frac{\varphi}{k} \text {, so } \lambda \leq \varphi=k \alpha \text {, and hence } K_{\kappa}^{\mathrm{sc}} \leq K_{\varepsilon}^{\mathrm{sc}}=K_{\alpha}^{\mathrm{sc}}=k .
$$

We remark that for simply connected hyperbolic plane regions we likewise obtain

$$
\tilde{\alpha}=\tilde{\varepsilon}=\frac{1}{k \delta} \text {, so } \lambda \leq \frac{2}{\delta}=2 k \tilde{\alpha} \text {, and hence } K_{\tilde{\kappa}}^{\mathrm{sc}} \leq K_{\tilde{\varepsilon}}^{\mathrm{sc}}=K_{\tilde{\alpha}}^{\mathrm{sc}}=2 k \text {. }
$$

We mention that Betsakos Bet08 has recently demonstrated that $K_{\tilde{\kappa}} \leq 8.27<2 k$.

A natural question to ask is whether we can improve our lower bounds if we consider only non-simply hyperbolic regions. The answer is that we cannot, even if we restrict attention to, say, $n$-connected regions for a fixed $n>1$. To see this, use kernel convergence ideas. 
5.C. Covering quasi-invariance. The hyperbolic metric is invariant under holomorphic coverings. One would expect that a good estimate for the hyperbolic metric might be quasi-invariant under coverings; this means it should not change by more than a fixed multiplicative factor under a covering of any region onto another. We corroborate this quasi-invariance with respect to coverings for all of the metrics studied in this article. In fact this is an immediate consequence of the following result which does not seem to have been previously documented.

We note in particular that the quasi-invariance of the Beardon-Pommerenke metric under coverings is not obvious at first glance since the manner in which the quantities $\delta_{\Omega}$ and $\beta_{\Omega}$ change under a covering is not so clear.

Proposition 5.5. Suppose we have a collection of conformal metrics $\rho(z)|d z|=$ $\rho_{\Omega}(z)|d z|$ defined for hyperbolic regions $\Omega \subset \hat{\mathbb{C}}$ all of which satisfy $\rho \leq \lambda \leq C \rho$ for some universal constant $C$. Let $f: \Omega \rightarrow \Omega^{\prime}$ be a holomorphic covering of hyperbolic regions in $\hat{\mathbb{C}}$. Then

$$
C^{-1} \rho(z)|d z| \leq f^{*}\left[\rho^{\prime}(w)|d w|\right] \leq C \rho(z)|d z| .
$$

Proof. The inequality $\lambda^{\prime} \leq C \rho^{\prime}$ yields

$$
\rho(z)|d z| \leq \lambda(z)|d z|=f^{*}\left[\lambda^{\prime}(w)|d w|\right] \leq C f^{*}\left[\rho^{\prime}(w)|d w|\right],
$$

and $\rho^{\prime} \leq \lambda^{\prime}$ provides

$$
f^{*}\left[\rho^{\prime}(w)|d w|\right] \leq f^{*}\left[\lambda^{\prime}(w)|d w|\right]=\lambda(z)|d z| \leq C \rho(z)|d z|
$$

as asserted.

5.D. Epilog. We conclude this article by asking some questions and making a conjecture.

\section{Questions.}

(a) What is $\tilde{\kappa}$ for $\mathbb{D}$ ?

(b) What are $\kappa$ and $\tilde{\kappa}$ for $\mathbb{D}^{*}$ ?

(c) What is $\kappa$ for an infinite strip?

(d) Does $\tilde{\alpha} \leq \alpha$ hold?

(e) What are the geodesics and isometries for these metrics?

We thank Alex Solynin for discussions regarding the following.

Conjecture. There is a homeomorphism $[1,+\infty) \stackrel{\vartheta}{\rightarrow}(\pi / 2,2 \pi / 3]$ (so $\vartheta(1)=2 \pi / 3)$ with the property that for all $t \geq 1$,

$$
\sup _{|a|=1=|b|} \lambda_{a b t}(0)=\lambda_{c \bar{c} t}(0) \quad \text { where } c=e^{i \vartheta(t)} .
$$

We mention that confirmation of the above would produce an answer for question (a) and possibly (b) and (c) too. To calculate $\tilde{\kappa}$ in $\mathbb{D}$, consider the Möbius change of variables $w=(z-r) /(1-r z)$ for a fixed $r \in(0,1)$. We see that determining $\tilde{\kappa}(r)$ in $\mathbb{D}$ reduces to finding $\sup _{|a|=1=|b|} \lambda_{a b t}(0)$ where $t=1 / r>1$. If we knew $\tilde{\kappa}$ in $\mathbb{D}$, we could confirm that there is a unique $t \in(0,1)$ with $\lambda_{01}(t)=\tilde{\kappa}_{\mathbb{D}}(t)$, and then 
(see Remark 4.9(b)) we would find that for $z \in \mathbb{D}^{*}$,

$$
\tilde{\kappa}_{\mathbb{D}^{*}}(z)= \begin{cases}\tilde{\kappa}_{\mathbb{D}}(z) & \text { when } t \leq|z|<1 \\ \lambda_{01}(|z|) & \text { when } 0<|z| \leq t,\end{cases}
$$

where $t \in(0,1)$ is the unique point with $\lambda_{01}(t)=\tilde{\kappa}_{\mathbb{D}}(t)$.

In order to obtain $\kappa$ in $\mathbb{D}^{*}$ we first recall from Remark 4.9(a) that in $\mathbb{D}^{*}$ we have $\kappa(z)=\sup _{|a|=1=|b|} \lambda_{a b 0}(z)$. A similar Möbius change of variables reduces this to determining the extremum $\sup _{|a|=1=|b|} \lambda_{a b t}(0)$ now for $t \in(0,1)$. This naturally leads to another conjecture. Finally, since we can identify $\mathbb{D}^{*}$ with $\mathbb{H} \backslash\{i\}$, knowledge of $\kappa$ in $\mathbb{D}^{*}$ would tell us $\kappa(i y)$ for $i y \in \mathbb{H} \backslash\{i\}$ (with $y \in(0,1)$ ) which in turn would yield $\kappa$ in an infinite strip.

\section{REFERENCES}

[Ahl73] L.V. Ahlfors, Conformal invariants: Topics in geometric function theory, McGraw-Hill, New York, 1973. MR0357743 (50:10211)

[Ahl79] Complex analysis: An introduction to the theory of analytic functions of one complex variable, third ed., McGraw-Hill, New York, 1979. MR0054016 (14:857a)

[Bet08] D. Betsakos, Estimation of the hyperbolic metric by using the punctured plane, Math. Z. (2008), to appear. MR2377748

[BP78] A.F. Beardon and Ch. Pommerenke, The Poincaré metric of plane domains, J. London Math. Soc. 18 (1978), no. 2, 475-483. MR.518232(80a:30020)

[Car60] C. Carathéodory, Theory of functions of a complex variable, $2^{\text {nd }}$ English ed., vol. 2, Chelsea Publ. Co., New York, 1960.

[Fer88] J. Ferrand, A characterization of quasiconformal mappings by the behavior of a function of three points, Complex Analysis, Joensuu 1987 (Berlin), Lecture Notes in Math., no. 1351, Springer-Verlag, 1988, pp. 110-123. MR982077 (89m:30040)

[GL01] F.P. Gardiner and N. Lakic, Comparing Poincaré densities, Ann. of Math. (2) 154 (2001), no. 2, 245-267. MR.1865971 (2003c:30046)

[Hei62] M. Heins, On a class of conformal metrics, Nagoya Math. J. 30 (1962), 1-60. MR0143901(26:1451)

[Hej74] D.A. Hejhal, Universal covering maps for variable regions, Math. Z 137 (1974), 7-20. MR0349989 (50:2482)

[Hem79] J.A. Hempel, The Poincaré metric on the twice punctured plane and the theorems of Landau and Schottky, J. London Math. Soc. 20 (1979), 435-445. MR561135|(81c:30025)

[HIM08] D.A. Herron, Z. Ibragimov, and D. Minda, Geodesics and curvature of Möbius invariant metrics, Rocky Mountain J. Math. (2008), vol. 38, no. 3, pp. 891-921.

[Jen81] J.A. Jenkins, On explicit bounds in Landau's theorem II, Canad. J. Math. 33 (1981), 559-562. MR627642 (83a:30026)

[Min82] D. Minda, Lower bounds for the hyperbolic metric in convex regions, Rocky Mountain J. Math. 12 (1982), 471-479. MR692577 (84j:30039)

[Min87] - Inequalities for the hyperbolic metric and applications to geometric function theory, Complex Analysis, I (College Park, MD, 1985-1986) (Berlin), Lecture Notes in Math., no. 1275, Springer-Verlag, 1987, pp. 235-252. MR922304 (89d:30029)

[Neh75] Z. Nehari, Conformal mapping, Dover Publ., Inc., New York, 1975. MR0377031 (51:13206)

[Pom79] Ch. Pommerenke, Uniformly perfect sets and the Poincaré metric, Arch. Math. (Basel) 32 (1979), no. 2, 192-199. MR534933 (80j:30073)

[Pom84] - On uniformly perfect sets and fuchsian groups, Analysis 4 (1984), no. 3-4, 299-321. MR 780609 (86e:30044)

[Sol97] A.Yu. Solynin, Functional inequalities via polarization, St. Petersburg Math. J. 8 (1997), 1015-1038. MR1458141 (98e:30001a)

[Sol99a] - Ordering of set, hyperbolic metrics, and harmonic measures, J. Math. Sci. 95 (1999), no. 3, 2256-2266. MR 1691288 (2000d:30068)

[Sol99b] _ Radial projection and the Poincaré metric, J. Math. Sci. 95 (1999), no. 3, 2267-2275. MR.1691289 (2000c:30086) 
[SV01] A.Yu. Solynin and M. Vuorinen, Estimates for the hyperbolic metric of the punctured plane and applications, Israel J. Math. 124 (2001), 29-60. MR.1856503 (2002j:30071)

[SV05] T. Sugawa and M. Vuorinen, Some inequalities for the Poincaré metric of plane domains, Mathematische Zeitschrift 250 (2005), no. 4, 885-906. MR2180380 (2006g:30075)

Department of Mathematical Sciences, 839 Old Chemistry Building, P.O. Box 210025, Cincinnati, Ohio 45221-0025

E-mail address: David.Herron@math.UC.edu

School of Integrated Studies, Pennsylvania College of Technology, Williamsport, Pennsylvania 17701

E-mail address: wma@pct.edu

Department of Mathematics, University of Cincinnati, Cincinnati, Ohio 45221

E-mail address: david.minda@math.uc.edu 